\title{
Quadrant marked mesh patterns in alternating permutations II
}

\author{
Sergey Kitaev and Jeffrey Remmel
}

This paper is a continuation of the systematic study of distribution of quadrant marked mesh patterns initiated in [7]. We study quadrant marked mesh patterns on up-down and down-up permutations.

\section{Introduction}

Let $\sigma=\sigma_{1} \cdots \sigma_{n}$ be a permutation in the symmetric group $S_{n}$ written in one-line notation. Then we will consider the graph of $\sigma, G(\sigma)$, to be the set of points $\left(i, \sigma_{i}\right)$ for $i=1, \ldots, n$. For example, the graph of the permutation $\sigma=471569283$ is pictured in Figure 1. Then if we draw a coordinate system centered at a point $\left(i, \sigma_{i}\right)$, we will be interested in the points that lie in the four quadrants I, II, III, and IV of that coordinate system as pictured in Figure 1.

For any $a, b, c, d \in \mathbb{N}$ where $\mathbb{N}=\{0,1,2, \ldots\}$ is the set of natural numbers and any $\sigma=\sigma_{1} \cdots \sigma_{n} \in S_{n}$, we say that $\sigma_{i}$ matches the quadrant marked mesh pattern $M M P(a, b, c, d)$ in $\sigma$ if in $G(\sigma)$ relative to the coordinate system which has the point $\left(i, \sigma_{i}\right)$ as its origin, there are $\geq a$ points in quadrant $\mathrm{I}, \geq b$ points in quadrant II, $\geq c$ points in quadrant III, and $\geq d$ points in quadrant IV. For example, if $\sigma=471569283$, the point $\sigma_{4}=5$ matches the quadrant marked mesh pattern $\operatorname{MMP}(2,1,2,1)$ since relative to the coordinate system with origin $(4,5)$, there are 3 points in $G(\sigma)$ in quadrant I, 1 point in $G(\sigma)$ in quadrant II, 2 points in $G(\sigma)$ in quadrant III, and 2 points in $G(\sigma)$ in quadrant IV.

Note that if a coordinate in $\operatorname{MMP}(a, b, c, d)$ is 0 , then there is no condition imposed on the points in the corresponding quadrant. In addition, we shall consider patterns $\operatorname{MMP}(a, b, c, d)$ where $a, b, c, d \in \mathbb{N} \cup\{\emptyset\}$. Here when one of the parameters $a, b, c$, or $d$ in $M M P(a, b, c, d)$ is the empty set, then for $\sigma_{i}$ to match $\operatorname{MMP}(a, b, c, d)$ in $\sigma=\sigma_{1} \cdots \sigma_{n} \in S_{n}$, it must be the case that there are no points in $G(\sigma)$ relative to coordinate system with

arXiv: 1205.0570 


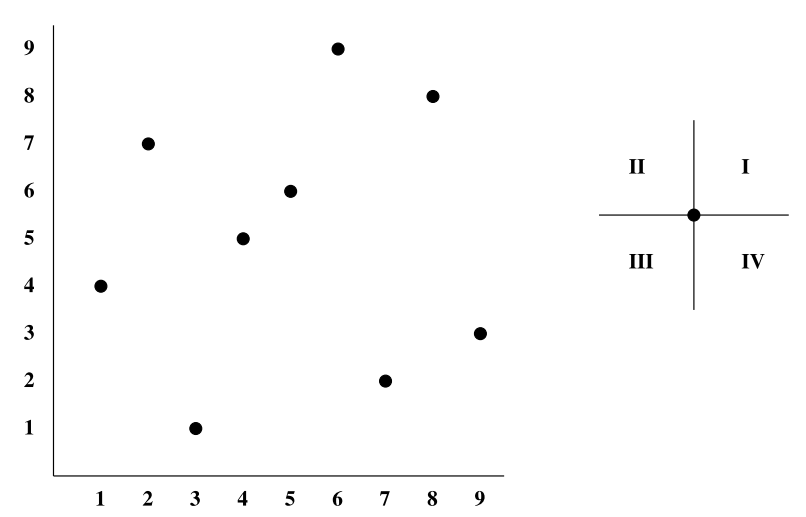

Figure 1: The graph of $\sigma=471569283$.

origin $\left(i, \sigma_{i}\right)$ in the corresponding quadrant. For example, if $\sigma=471569283$, the point $\sigma_{3}=1$ matches the marked mesh pattern $\operatorname{MMP}(4,2, \emptyset, \emptyset)$ since relative to the coordinate system with origin $(3,1)$, there are 6 points in $G(\sigma)$ in quadrant I, 2 points in $G(\sigma)$ in quadrant II, no points in $G(\sigma)$ in quadrant III, and no points in $G(\sigma)$ in quadrant IV. We let $\operatorname{mmp}^{(a, b, c, d)}(\sigma)$ denote the number of $i$ such that $\sigma_{i}$ matches the marked mesh pattern $M M P(a, b, c, d)$ in $\sigma$.

The notion of mesh patterns was introduced by Brändén and Claesson [4] to provide explicit expansions for certain permutation statistics as, possibly infinite, linear combinations of (classical) permutation patterns (see [6] for a comprehensive introduction to the theory of permutation patterns). This notion was further studied in [3, 5, 7-11, 14].

Note how the (two-dimensional) notation of Úlfarsson [14] for marked mesh patterns corresponds to our (one-line) notation for quadrant marked mesh patterns. For example,

$$
\begin{aligned}
& \operatorname{MMP}(0,0, k, 0)=\frac{}{k}, \quad \operatorname{MMP}(k, 0,0,0)=\longdiv { \sqrt { k } },
\end{aligned}
$$

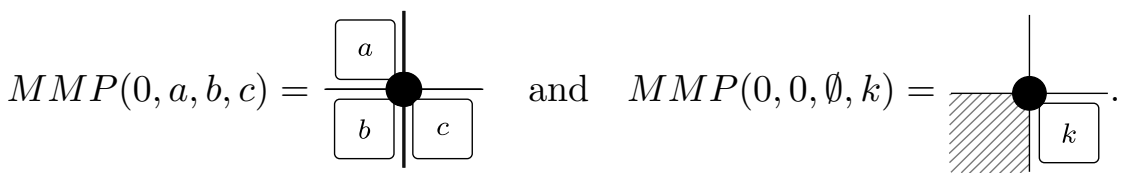

Kitaev and Remmel [7] studied the distribution of quadrant marked mesh patterns in the symmetric group $S_{n}$, and Kitaev, Remmel, and Tiefenbruck $[9,10]$ studied the distribution of quadrant marked mesh patterns 
in 132-avoiding permutations in $S_{n}$. In [8], Kitaev and Remmel studied the distribution of the statistics $\mathrm{mmp}^{(1,0,0,0)}, \mathrm{mmp}^{(0,1,0,0)}, \mathrm{mmp}^{(0,0,1,0)}$, and $\mathrm{mmp}^{(0,0,0,1)}$ in the set of up-down and down-up permutations. The main goal of this paper is to study the distribution of the statistics $\mathrm{mmp}^{(1,0, \emptyset, 0)}$, $\mathrm{mmp}^{(0,1,0, \emptyset)}, \mathrm{mmp}^{(0, \emptyset, 0,1)}$, and $\mathrm{mmp} \mathrm{p}^{(\emptyset, 0,1,0)}$ in the set of up-down and downup permutations. Given a permutation $\sigma=\sigma_{1} \cdots \sigma_{n} \in S_{n}$, we let $\operatorname{Des}(\sigma)=$ $\left\{i: \sigma_{i}>\sigma_{i+1}\right\}$. Then we say that $\sigma$ is an up-down permutation if $\operatorname{Des}(\sigma)$ is the set of all even numbers less than or equal to $n$ and a down-up permutation if $\operatorname{Des}(\sigma)$ is the set of all odd numbers less than or equal to $n$. That is, $\sigma$ is an up-down permutation if

$$
\sigma_{1}<\sigma_{2}>\sigma_{3}<\sigma_{4}>\sigma_{5}<\cdots
$$

and $\sigma$ is an down-up permutation if

$$
\sigma_{1}>\sigma_{2}<\sigma_{3}>\sigma_{4}<\sigma_{5}>\cdots .
$$

Let $U D_{n}$ denote the set of all up-down permutations in $S_{n}$ and $D U_{n}$ denote the set of all down-up permutations in $S_{n}$. Given a permutation $\sigma=$ $\sigma_{1} \cdots \sigma_{n} \in S_{n}$, we define the reverse of $\sigma, \sigma^{r}$, to be $\sigma_{n} \sigma_{n-1} \cdots \sigma_{2} \sigma_{1}$ and the complement of $\sigma, \sigma^{c}$, to be $\left(n+1-\sigma_{1}\right) \cdots\left(n+1-\sigma_{n}\right)$.

For $n \geq 1$, we let

$$
\begin{aligned}
& A_{2 n}^{(a, b, c, d)}(x)=\sum_{\sigma \in U D_{2 n}} x^{\mathrm{mmp}^{(a, b, c, d)}(\sigma)}, \quad B_{2 n-1}^{(a, b, c, d)}(x)=\sum_{\sigma \in U D_{2 n-1}} x^{\mathrm{mmp}^{(a, b, c, d)}(\sigma)}, \\
& C_{2 n}^{(a, b, c, d)}(x)=\sum_{\sigma \in D U_{2 n}} x^{\mathrm{mmp}^{(a, b, d, d)}(\sigma)}, \text { and } D_{2 n-1}^{(a, b, c, d)}(x)=\sum_{\sigma \in D U_{2 n-1}} x^{\mathrm{mmp}^{(a, b, c, d)}(\sigma)} .
\end{aligned}
$$

We then have the following simple proposition.

Proposition 1. For all $n \geq 1$,

(1) $A_{2 n}^{(a, b, c, d)}(x)=C_{2 n}^{(b, a, d, c)}(x)=C_{2 n}^{(d, c, b, a)}(x)=A_{2 n}^{(c, d, a, b)}(x)$,

(2) $C_{2 n}^{(a, b, c, d)}(x)=A_{2 n}^{(b, a, d, c)}(x)=A_{2 n}^{(d, c, b, a)}(x)=C_{2 n}^{(c, d, a, b)}(x)$,

(3) $B_{2 n-1}^{(a, b, c, d)}(x)=B_{2 n-1}^{(b, a, d, c)}(x)=D_{2 n-1}^{(d, c, b, a)}(x)=D_{2 n-1}^{(c, d, a, b)}(x)$, and

(4) $D_{2 n-1}^{(a, b, c, d)}(x)=D_{2 n-1}^{(b, a, d, c)}(x)=B_{2 n-1}^{(d, c, b, a)}(x)=B_{2 n-1}^{(c, d, a, b)}(x)$.

Proof. It is easy to see that for any $\sigma \in S_{n}$,

$$
\mathrm{mmp}^{(a, b, c, d)}(\sigma)=\mathrm{mmp}^{(b, a, d, c)}\left(\sigma^{r}\right)=\mathrm{mmp}^{(d, c, b, a)}\left(\sigma^{c}\right)=\mathrm{mmp}^{(c, d, a, b)}\left(\left(\sigma^{r}\right)^{c}\right) .
$$


Then part 1 easily follows since

$$
\sigma \in U D_{2 n} \Longleftrightarrow \sigma^{r} \in D U_{2 n} \Longleftrightarrow \sigma^{c} \in D U_{2 n} \Longleftrightarrow\left(\sigma^{r}\right)^{c} \in U D_{2 n} .
$$

Parts 2, 3, and 4 are proved in a similar manner.

In [8], we studied the distribution of the statistics $\operatorname{mmp}^{(1,0,0,0)}$, $m m p^{(0,1,0,0)}, \mathrm{mmp}^{(0,0,1,0)}$, and $m \mathrm{mp}^{(0,0,0,1)}$ in the set of up-down and downup permutations. It follows from Proposition 1 that the study of the distribution of the statistics $m m p^{(1,0,0,0)}, \mathrm{mmp}^{(0,1,0,0)}, \mathrm{mmp}^{(0,0,1,0)}$, and $\mathrm{mmp}^{(0,0,0,1)}$ in the set of up-down and down-up permutations can be reduced to the study of the following generating functions:

$$
\begin{aligned}
& A^{(1,0,0,0)}(t, x)=1+\sum_{n \geq 1} A_{2 n}^{(1,0,0,0)}(x) \frac{t^{2 n}}{(2 n) !}, \\
& B^{(1,0,0,0)}(t, x)=\sum_{n \geq 1} B_{2 n-1}^{(1,0,0,0)}(x) \frac{t^{2 n-1}}{(2 n-1) !}, \\
& C^{(1,0,0,0)}(t, x)=1+\sum_{n \geq 1} C_{2 n}^{(1,0,0,0)}(x) \frac{t^{2 n}}{(2 n) !}, \text { and } \\
& D^{(1,0,0,0)}(t, x)=\sum_{n \geq 1} D_{2 n-1}^{(1,0,0,0)}(x) \frac{t^{2 n-1}}{(2 n-1) !} .
\end{aligned}
$$

In the case when $x=1$, these generating functions are well known. That is, for any $(a, b, c, d)$, let $A_{2 n}(1)=A_{2 n}^{(a, b, c, d)}(1), B_{2 n+1}(1)=B_{2 n+1}^{(a, b, c, d)}(1)$, $C_{2 n}(1)=C_{2 n}^{(a, b, c, d)}(1)$, and $D_{2 n}(1)=D_{2 n}^{(a, b, c, d)}(1)$. The operation of complementation shows that $A_{2 n}(1)=C_{2 n}(1)$ and $B_{2 n-1}(1)=D_{2 n-1}(1)$ for all $n \geq 1$. André $[1,2]$ proved that

$$
1+\sum_{n \geq 0} A_{2 n}(1) \frac{t^{2 n}}{(2 n) !}=\sec (t)
$$

and

$$
\sum_{n \geq 1} B_{2 n-1}(1) \frac{t^{2 n+1}}{(2 n+1) !}=\tan (t)
$$

In [8], we proved the following which can be viewed as a refinement of André's results. 


\section{Theorem 1.}

(6) $v D^{(1,0,0,0)}(t, x)=\int_{0}^{t}(\sec (x z))^{1+\frac{1}{x}} d z$.

In this paper, we prove a different refinement of André's results by studying the distribution of the statistics $m m p^{(1,0, \emptyset, 0)}, \operatorname{mmp}^{(\emptyset, 0,1,0)}, \operatorname{mmp}^{(0,1,0, \emptyset)}$, and $\mathrm{mmp}^{(0, \emptyset, 0,1)}$ in the set of up-down and down-up permutations. It follows from Proposition 1 that the study of the distribution of the statistics $m m p^{(1,0, \emptyset, 0)}, \mathrm{mmp}^{(\emptyset, 0,1,0)}, \mathrm{mmp}^{(0,1,0, \emptyset)}$, and $\mathrm{mmp} \mathrm{p}^{(0, \emptyset, 0,1)}$ in the set of up-down and down-up permutations can be reduced to the study of the following generating functions:

$$
\begin{aligned}
& A^{(1,0, \emptyset, 0)}(t, x)=1+\sum_{n \geq 1} A_{2 n}^{(1,0, \emptyset, 0)}(x) \frac{t^{2 n}}{(2 n) !}, \\
& B^{(1,0, \emptyset, 0)}(t, x)=\sum_{n \geq 1} B_{2 n-1}^{(1,0, \emptyset, 0)}(x) \frac{t^{2 n-1}}{(2 n-1) !}, \\
& C^{(1,0, \emptyset, 0)}(t, x)=1+\sum_{n \geq 1} C_{2 n}^{(1,0, \emptyset, 0)}(x) \frac{t^{2 n}}{(2 n) !}, \text { and } \\
& D^{(1,0, \emptyset, 0)}(t, x)=\sum_{n \geq 1} D_{2 n-1}^{(1,0, \emptyset, 0)}(x) \frac{t^{2 n-1}}{(2 n-1) !} .
\end{aligned}
$$

The main goal of this paper is prove the following theorem.

\section{Theorem 2.}

$$
\begin{aligned}
A^{(1,0, \emptyset, 0)}(t, x)= & (\sec (t))^{x} \\
B^{(1,0, \emptyset, 0)}(t, x)= & \frac{\sin (t) \cos (t)(1-x+x \sec (t))}{x+(1-x) \cos (t)} \\
& \times\left((1-x)_{2} F_{1}\left(\frac{1}{2}, \frac{1+x}{2} ; \frac{3}{2} ;(\sin (t))^{2}\right)\right. \\
& \left.+x_{2} F_{1}\left(\frac{1}{2}, \frac{2+x}{2} ; \frac{3}{2} ;(\sin (t))^{2}\right)\right),
\end{aligned}
$$




$$
\begin{aligned}
D^{(1,0, \emptyset, 0)}(t, x)= & x(\sec (t))^{x} \int_{0}^{t}(\cos (z))^{x} d z+(1-x) \int_{0}^{t}(\sec (z))^{x} d z, \text { and } \\
C^{(1,0, \emptyset, 0)}(t, x)= & 1+\int_{0}^{t}\left[x(\sec (z))^{x}(1-x+x \sec (z)) \int_{0}^{z} \cos (y) d y\right] d z \\
& +(1-x) \int_{0}^{t} B^{(1,0, \emptyset, 0)}(t, z) d z
\end{aligned}
$$

Here ${ }_{2} F_{1}(a, b ; c ; z)=\sum_{n=0}^{\infty} \frac{(a)_{n}(b)_{n}}{(c)_{n}} \frac{z^{n}}{n !}$ where $(x)_{n}=x(x-1) \cdots(x-n+1)$ if $n \geq 1$ and $(x)_{0}=1$.

One can use these generating functions to find some initial values of the polynomials $A_{2 n}^{(1,0, \emptyset, 0)}(x), B_{2 n-1}^{(1,0, \emptyset, 0)}(x), C_{2 n}^{(1,0, \emptyset, 0)}(x)$, and $D_{2 n-1}^{(1,0, \emptyset, 0)}(x)$. For example, we have used Mathematica to compute the following tables.

\begin{tabular}{|l|l|}
\hline$n$ & $A_{2 n}^{(1,0, \emptyset, 0)}(x)$ \\
\hline 0 & 1 \\
\hline 1 & $x$ \\
\hline 2 & $x(2+3 x)$ \\
\hline 3 & $x\left(16+30 x+15 x^{2}\right)$ \\
\hline 4 & $x\left(272+588 x+420 x^{2}+105 x^{3}\right)$ \\
\hline 5 & $x\left(7936+18960 x+16380 x^{2}+6300 x^{3}+945 x^{4}\right)$ \\
\hline 6 & $x\left(353792+911328 x+893640 x^{2}+429660 x^{3}+103950 x^{4}+10395 x^{5}\right)$ \\
\hline
\end{tabular}

\begin{tabular}{|l|l|}
\hline$n$ & $B_{2 n+1}^{(1,0, \emptyset, 0)}(x)$ \\
\hline 0 & 1 \\
\hline 1 & $2 x$ \\
\hline 2 & $x(7+9 x)$ \\
\hline 3 & $x\left(77+135 x+60 x^{2}\right)$ \\
\hline 4 & $x\left(1657+3444 x+2310 x^{2}+525 x^{3}\right)$ \\
\hline 5 & $x\left(58457+135945 x+112770 x^{2}+40950 x^{3}+5670 x^{4}\right)$ \\
\hline 6 & $x\left(3056557+7715664 x+7347945 x^{2}+3395700 x^{3}+777625 x^{4}\right.$ \\
& $\left.+72765 x^{5}\right)$ \\
\hline
\end{tabular}

\begin{tabular}{|l|l|}
\hline$n$ & $C_{2 n}^{(1,0, \emptyset, 0)}(x)$ \\
\hline 0 & 1 \\
\hline 1 & 1 \\
\hline 2 & $x(2+3 x)$ \\
\hline 3 & $x\left(7+35 x+19 x^{2}\right)$ \\
\hline 4 & $x\left(77+581 x+571 x^{2}+156 x^{3}\right)$ \\
\hline 5 & $x\left(1657+16428 x+21066 x^{2}+9738 x^{3}+1587 x^{4}\right)$ \\
\hline 6 & $x\left(58457+712579 x+1079747 x^{2}+652452 x^{3}+180240 x^{4}+19290 x^{5}\right)$ \\
\hline
\end{tabular}




\begin{tabular}{|l|l|}
\hline$n$ & $D_{2 n+1}^{(1,0, \emptyset, 0)}(x)$ \\
\hline 0 & 1 \\
\hline 1 & $x(1+x)$ \\
\hline 2 & $x\left(2+9 x+5 x^{2}\right)$ \\
\hline 3 & $x\left(16+110 x+113 x^{2}+33 x^{3}\right)$ \\
\hline 4 & $x\left(272+2492 x+3288 x^{2}+1605 x^{3}+279 x^{4}\right)$ \\
\hline 5 & $x\left(7936+90384 x+139756 x^{2}+87456 x^{3}+25365 x^{4}+2895 x^{5}\right)$ \\
\hline 6 & $x\left(353792+4803040 x+8323816 x^{2}+6110100 x^{3}+2297778 x^{4}\right.$ \\
& $\left.+444045 x^{5}+35685 x^{6}\right)$ \\
\hline
\end{tabular}

The outline of this paper is as follows. In Section 2, we shall prove Theorem 2. Then in Section 3, we shall show how several of the entries of the tables above can be explained. In particular, we will derive formulas for the coefficient of the highest and lowest coefficient of $x$ in the polynomials the polynomials $A_{2 n}^{(1,0, \emptyset, 0)}(x), B_{2 n+1}^{(1,0, \emptyset, 0)}(x), C_{2 n}^{(1,0, \emptyset, 0)}(x)$, and $D_{2 n+1}^{(1,0, \emptyset, 0)}(x)$, as well as formulas for the second highest and second lowest coefficient of $x$ in these polynomials. Finally, in Section 4, we shall discuss some connections with our previous work [8] on quadrant marked mesh patterns in alternating permutations, as well as some directions for further research.

In this paper, we need the following notation. Given a sequence $\sigma=$ $\sigma_{1} \cdots \sigma_{n}$ of distinct integers, we let $\operatorname{red}(\sigma)$ be the permutation found by replacing the $i$ th largest integer that appears in $\sigma$ by $i$. For example, if $\sigma=2854$, then $\operatorname{red}(\sigma)=1432$.

\section{Proof of Theorem 2}

The proof of all parts of Theorem 2 proceed in the same manner. That is, there are simple recursions satisfied by the polynomials $A_{2 n}^{(1,0, \emptyset, 0)}(x)$, $B_{2 n+1}^{(1,0, \emptyset, 0)}(x), C_{2 n}^{(1,0, \emptyset, 0)}(x)$, and $D_{2 n+1}^{(1,0, \emptyset, 0)}(x)$ based on the possible positions of 1 in an up-down or a down-up permutation.

\subsection{The generating function $A^{(1,0, \emptyset, 0)}(t, x)$}

If $\sigma=\sigma_{1} \cdots \sigma_{2 n} \in U D_{2 n}$, then 1 must occur in one of the positions $1,3, \ldots$, $2 n-1$. Let $U D_{2 n}^{(2 k+1)}$ denote the set of permutations $\sigma \in U D_{2 n}$ such that $\sigma_{2 k+1}=1$. A schematic diagram of an element in $U D_{2 n}^{(2 k+1)}$ is pictured in Figure 2 .

Consider a $\sigma=\sigma_{1} \cdots \sigma_{2 n} \in U D_{2 n}^{(2 k+1)}$ where $0 \leq k \leq n-1$. Note that there are $\left(\begin{array}{c}2 n-1 \\ 2 k\end{array}\right)$ ways to pick the elements which occur to the right of posi- 


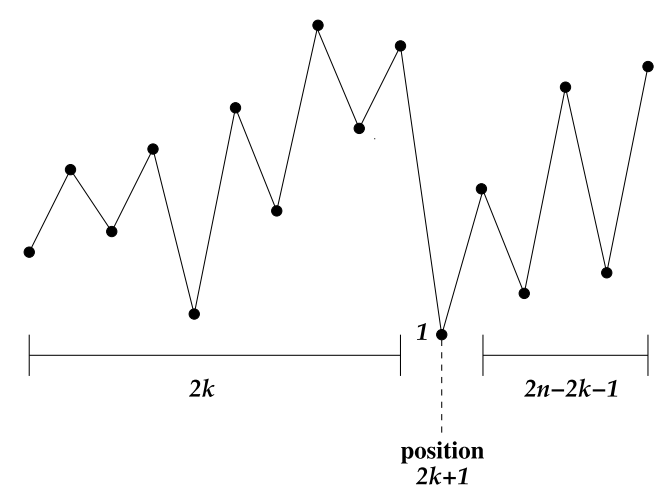

Figure 2: The graph of a $\sigma \in U D_{2 n}^{(2 k+1)}$.

tion $2 k+1$ in such a $\sigma$ and there are $D_{2 n-2 k-1}(1)=B_{2 n-2 k-1}(1)$ ways to order them since the elements to the right of position $2 k+1$ must form a downup permutation of length $2 n-2 k-1$. The fact that $\sigma_{2 k+1}=1$ implies that $\sigma_{2 k+1}$ matches $M M P(1,0, \emptyset, 0)$ in $\sigma$ and that none of the elements to the right of position $2 k+1$ match $\operatorname{MMP}(1,0, \emptyset, 0)$ in $\sigma$. Thus, the contribution of the elements to the right of position $2 k+1$ in $\sum_{\sigma \in U D_{2 n}^{(2 k+1)}} x^{\operatorname{mmp}^{(1,0, \emptyset, 0)}(\sigma)}$ is $B_{2 n-2 k-1}(1)$. Now the only possible elements of $\sigma_{1}, \ldots, \sigma_{2 k}$ that can contribute to $\operatorname{mmp}^{(1,0, \emptyset, 0)}(\sigma)$ are $\sigma_{1}, \sigma_{3}, \ldots, \sigma_{2 k-1}$. Since each of the elements have an element to its right in $\sigma_{1} \cdots \sigma_{2 k}$ which is larger than that element, it follows that the elements to the right of position $2 k+1$ have no effect on whether $\sigma_{1}, \ldots, \sigma_{2 k}$ can contribute to $\operatorname{mmp}^{(1,0, \emptyset, 0)}(\sigma)$. Hence, the contribution of the elements to the left of position $2 k+1$ in $\sum_{\sigma \in U D_{2 n}^{(2 k+1)}} x^{\mathrm{mmp}^{(1,0, \emptyset, 0)}(\sigma)}$ is $A_{2 k}^{(1,0, \emptyset, 0)}(x)$. It thus follows that for $n \geq 1$,

$$
A_{2 n}^{(1,0, \emptyset, 0)}(x)=x \sum_{k=0}^{n-1}\left(\begin{array}{c}
2 n-1 \\
2 k
\end{array}\right) B_{2 n-2 k-1}(1) A_{2 k}^{(1,0, \emptyset, 0)}(x)
$$

or, equivalently,

$$
\frac{A_{2 n}^{(1,0, \emptyset, 0)}(x)}{(2 n-1) !}=x \sum_{k=0}^{n-1} \frac{B_{2 n-2 k-1}(1)}{(2 n-2 k-1) !} \frac{A_{2 k}^{(1,0, \emptyset, 0)}(x)}{(2 k) !} .
$$

Multiplying both sides of (7) by $t^{2 n-1}$ and summing for $n \geq 1$, we see that

$$
\sum_{n \geq 1} \frac{A_{2 n}^{(1,0, \emptyset, 0)}(x) t^{2 n-1}}{(2 n-1) !}=x\left(\sum_{k \geq 1} \frac{B_{2 k-1}(1) t^{2 k-1}}{(2 k-1) !}\right)\left(\sum_{k \geq 0} \frac{A_{2 k}^{(1,0, \emptyset, 0)}(x) t^{2 k}}{(2 k) !}\right) .
$$


By (2),

$$
\sum_{k \geq 1} \frac{B_{2 k-1}(1) t^{2 k-1}}{(2 k-1) !}=\tan (t)
$$

so that

$$
\frac{\partial}{\partial t} A^{(1,0, \emptyset, 0)}(t, x)=x \tan (t) A^{(1,0, \emptyset, 0)}(t, x) .
$$

Our initial condition is that $A^{(1,0, \emptyset, 0)}(0, x)=1$. It is easy to check that the solution to this differential equation is

$$
A^{(1,0, \emptyset, 0)}(t, x)=(\sec (t))^{x} .
$$

\subsection{The generating function $B^{(1,0, \emptyset, 0)}(t, x)$}

If $\sigma=\sigma_{1} \cdots \sigma_{2 n+1} \in U D_{2 n+1}$, then 1 must occur in one of the positions $1,3, \ldots, 2 n+1$. Let $U D_{2 n+1}^{(2 k+1)}$ denote the set of permutations $\sigma \in U D_{2 n+1}$ such that $\sigma_{2 k+1}=1$. A schematic diagram of an element in $U D_{2 n}^{(2 k+1)}$ is pictured in Figure 3.

A permutation $\sigma=\sigma_{1} \cdots \sigma_{2 n+1} \in U D_{2 n+1}^{(2 n+1)}$ ends with 1 so that $\sigma_{2 n+1}=$ 1 does not match $M M P(1,0, \emptyset, 0)$ in $\sigma$. Moreover, $\operatorname{red}\left(\sigma_{1} \cdots \sigma_{2 n}\right) \in U D_{2 n}$ and $\sigma_{2 n+1}=1$ cannot affect whether any of the other elements in $\sigma$ match $M M P(1,0, \emptyset, 0)$. Thus,

$$
\sum_{\sigma \in U D_{2 n+1}^{(2 n+1)}} x^{\mathrm{mmp}^{(1,0, \emptyset, 0)}(\sigma)}=A_{2 n}^{(1,0, \emptyset, 0)}(x) .
$$

Next, consider $U D_{2 n+1}^{(2 k+1)}$ where $0 \leq k \leq n-1$. Note that there are $\left(\begin{array}{c}2 n \\ 2 k\end{array}\right)$ ways to pick the elements which occur to the right of position $2 k+1$ in such a $\sigma$ and there are $C_{2 n-2 k}(1)=A_{2 n-2 k}(1)$ ways to order them since the elements to the right of position $2 k+1$ form a down-up permutation of length $2 n-2 k$. That is, the fact that $\sigma_{2 k+1}=1$ implies that $\sigma_{2 k+1}$ matches $\operatorname{MMP}(1,0, \emptyset, 0)$ in $\sigma$ and that none of the elements to the right of position $2 k+1$ matches $\operatorname{MMP}(1,0, \emptyset, 0)$ in $\sigma$. Thus, the contribution of the elements to the right of position $2 k+1$ in $\sum_{\sigma \in U D_{2 n+1}^{(2 k+1)}} x^{\mathrm{mmp}^{(1,0, \emptyset, 0)}(\sigma)}$ is $C_{2 n-2 k}(1)=$ $A_{2 n-2 k}(1)$ since the elements to the right of position $2 k+1$ must form a downup permutation of length $2 n-2 k$. As we proved above, the elements to the right of position $2 k+1$ have no effect on whether $\sigma_{1}, \ldots, \sigma_{2 k}$ can contribute to $\mathrm{mmp}^{(1,0, \emptyset, 0)}(\sigma)$. It follows that the contribution of the elements to the 


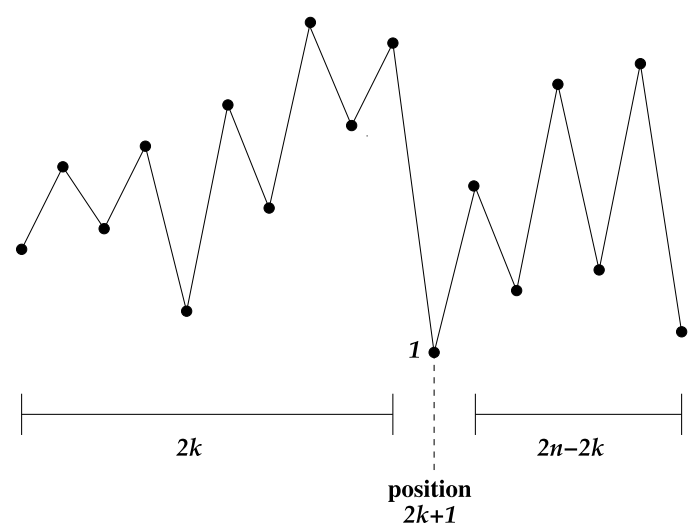

Figure 3: The graph of a $\sigma \in U D_{2 n+1}^{(2 k+1)}$.

left of position $2 k+1$ in $\sum_{\sigma \in U D_{2 n+1}^{(2 k+1)}} x^{\mathrm{mmp}^{(1,0, \emptyset, 0)}(\sigma)}$ is $A_{2 k}^{(1,0, \emptyset, 0)}(x)$. It thus follows that for $n \geq 1$,

$$
B_{2 n+1}^{(1,0, \emptyset, 0)}(x)=A_{2 n}^{(1,0, \emptyset, 0)}(x)+x \sum_{k=0}^{n-1}\left(\begin{array}{l}
2 n \\
2 k
\end{array}\right) A_{2 n-2 k}(1) A_{2 k}^{(1,0, \emptyset, 0)}(x)
$$

or, equivalently,

$$
\frac{B_{2 n+1}^{(1,0, \emptyset, 0)}(x)}{(2 n) !}=\frac{A_{2 n}^{(1,0, \emptyset, 0)}(x)}{(2 n) !}+x \sum_{k=0}^{n-1} \frac{A_{2 n-2 k}(1)}{(2 n-2 k) !} \frac{A_{2 k}^{(1,0, \emptyset, 0)}(x)}{(2 k) !} .
$$

Note that $B_{1}^{(1,0, \emptyset, 0)}(x)=1$. Multiplying both sides of (9) by $t^{2 n}$ and summing for $n \geq 1$, we see that

$$
\begin{aligned}
& \sum_{n \geq 0} \frac{B_{2 n+1}^{(1,0, \emptyset, 0)}(x) t^{2 n}}{(2 n) !} \\
& \quad=\sum_{n \geq 0} \frac{A_{2 n}^{(1,0, \emptyset, 0)}(x) t^{2 n}}{(2 n) !}+x\left(\sum_{k \geq 1} \frac{A_{2 k}(1) t^{2 k}}{(2 k) !}\right)\left(\sum_{k \geq 0} \frac{A_{2 k}^{(1,0, \emptyset, 0)}(x) t^{2 k}}{(2 k) !}\right)
\end{aligned}
$$

By (1),

$$
\sum_{k \geq 0} \frac{A_{2 k}(1) t^{2 k}}{(2 k) !}=\sec (t)
$$


so that

$$
\left.\frac{\partial}{\partial t} B^{(1,0, \emptyset, 0)}(t, x)=(\sec (t))^{x}+x(\sec (t))^{x}(\sec (t)-1)\right)
$$

Thus,

$$
\frac{\partial}{\partial t} B^{(1,0, \emptyset, 0)}(t, x)=(\sec (t))^{x}(1-x+x \sec (t)) .
$$

Our initial condition is that $B^{(1,0, \emptyset, 0)}(0, x)=0$. We used Mathematica to solve this differential equation which gave the following formula for $B^{(1,0, \emptyset, 0)}(t, x)$ :

$$
\begin{aligned}
B^{(1,0, \emptyset, 0)}(t, x)= & \frac{\sin (t) \cos (t)(1-x+x \sec (t))}{x+(1-x) \cos (t)} \\
& \times\left((1-x){ }_{2} F_{1}\left(\frac{1}{2}, \frac{1+x}{2} ; \frac{3}{2} ;(\sin (t))^{2}\right)\right. \\
& \left.+x_{2} F_{1}\left(\frac{1}{2}, \frac{2+x}{2} ; \frac{3}{2} ;(\sin (t))^{2}\right)\right) .
\end{aligned}
$$

\subsection{The generating function $D^{(1,0, \emptyset, 0)}(t, x)$}

If $\sigma=\sigma_{1} \cdots \sigma_{2 n+1} \in D U_{2 n+1}$, then 1 must occur in one of the positions $2,4, \ldots, 2 n$. Let $D U_{2 n+1}^{(2 k)}$ denote the set of permutations $\sigma \in D U_{2 n+1}$ such that $\sigma_{2 k}=1$. A schematic diagram of an element in $D U_{2 n+1}^{(2 k)}$ is pictured in Figure 4 .

Let

$$
D_{2 n+1}^{(1,0, \emptyset, 0)}(x, y)=\sum_{\sigma \in D U_{2 n+1}} x^{\mathrm{mmp}^{(1,0, \emptyset, 0)}(\sigma)} y^{\chi\left(\sigma_{1}=2 n+1\right)} .
$$

First, we want to study the polynomial $\bar{D}_{2 n+1}^{(1,0, \emptyset, 0)}(x)=D_{2 n+1}^{(1,0, \emptyset, 0)}(x, x)$. Suppose that $\sigma=\sigma_{1} \cdots \sigma_{2 n+1}$ is an element of $D U_{2 n+1}$. If $\sigma_{1}<2 n+1$, then $\sigma_{1}$ will automatically contribute to $\mathrm{mmp}^{(1,0, \emptyset, 0)}(\sigma)$. However, if $\sigma_{1}=2 n+1$, then $\sigma_{1}$ will not contribute to $\mathrm{mmp}^{(1,0, \emptyset, 0)}(\sigma)$. Thus, the difference between $\bar{D}_{2 n+1}^{(1,0, \emptyset, 0)}(x)$ and $D_{2 n+1}^{(1,0, \emptyset, 0)}(x)$ is that $\sigma_{1}$ always contributes a factor of $x$ to $x^{\mathrm{mmp}^{(1,0, \emptyset, 0)}(\sigma)} x^{\chi\left(\sigma_{1}=2 n+1\right)}$.

First, we shall prove a simple recursion for $\bar{D}_{2 n+1}^{(1,0, \emptyset, 0)}(x)$. That is, consider a $\sigma=\sigma_{1} \cdots \sigma_{2 n+1} \in D U_{2 n+1}^{(2 k)}$ where $1 \leq k \leq n$. Note that there are $\left(\begin{array}{c}2 n \\ 2 k-1\end{array}\right)$ ways to pick the elements which occur to the right of position 


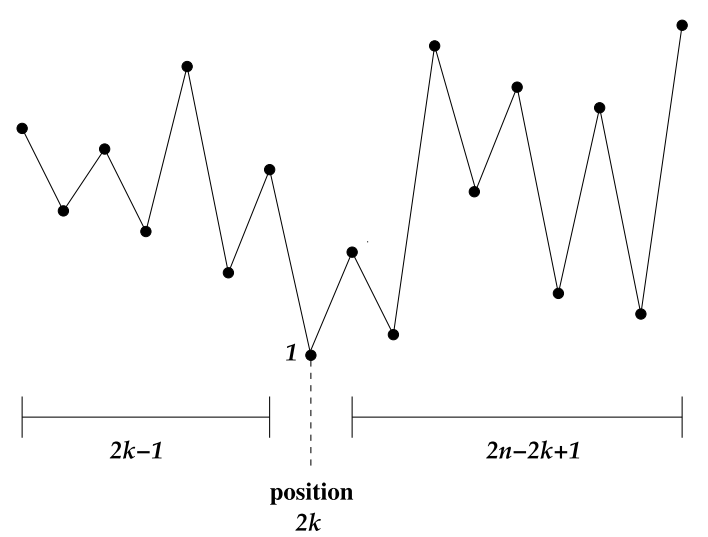

Figure 4: The graph of a $\sigma \in D U_{2 n+1}^{(2 k)}$.

$2 k$ in such a $\sigma$ and there are $D_{2 n-2 k+1}(1)=B_{2 n-2 k-1}(1)$ ways to order them since the elements to the right of position $2 k$ form a down-up permutation of length $2 n-2 k+1$. For a $\sigma=\sigma_{1} \cdots \sigma_{2 n+1} \in D U_{2 n+1}^{(2 k)}$, none of the elements $\sigma_{i}$ for $i>2 k$ matches $M M P(1,0, \emptyset, 0)$ in $\sigma$ and $\sigma_{2 k}=1$ always matches $\operatorname{MMP}(1,0, \emptyset, 0)$ in $\sigma$. Thus, the only other elements of $\sigma$ that can possibly contribute to $\operatorname{mmp}^{(1,0, \emptyset, 0)}(\sigma) x^{\chi\left(\sigma_{1}=2 n+1\right)}$ are the elements $\sigma_{1}, \sigma_{2}, \sigma_{4}, \ldots, \sigma_{2 k-2}$. Since in $\bar{D}_{2 n+1}^{(1,0, \emptyset, 0)}(x), \sigma_{1}$ always contributes to $\operatorname{mmp}^{(1,0, \emptyset, 0)}(\sigma) x^{\chi\left(\sigma_{1}=2 n+1\right)}$ and the elements to the right of position $2 k$ have no effect on whether $\sigma_{2}, \ldots, \sigma_{2 k-2}$ contribute to $\operatorname{mmp}^{(1,0, \emptyset, 0)}(\sigma)$, it follows that the contribution of the elements to the left of position $2 k$ to

$$
\sum_{\sigma \in D U_{2 n+1}^{(2 k)}} x^{\mathrm{mmp}^{(1,0, \emptyset, 0)}(\sigma)} x^{\chi\left(\sigma_{1}=2 n+1\right)}
$$

is $\bar{D}_{2 k-1}(x)$. Hence, for $n \geq 1$,

$$
\bar{D}_{2 n+1}^{(1,0, \emptyset, 0)}(x)=x \sum_{k=1}^{n}\left(\begin{array}{c}
2 n \\
2 k-1
\end{array}\right) \bar{D}_{2 k-1}^{(1,0, \emptyset, 0)}(x) B_{2 n-2 k+1}(1)
$$

or, equivalently,

$$
\frac{\bar{D}_{2 n+1}^{(1,0, \emptyset, 0)}(x)}{(2 n) !}=x \sum_{k=1}^{n} \frac{\bar{D}_{2 k-1}^{(1,0, \emptyset, 0)}(x)}{(2 k-1) !} \frac{B_{2 n-2 k+1}(1)}{(2 n-2 k+1) !} .
$$


Note that $\bar{D}_{1}^{(1,0, \emptyset, 0)}(x)=x$. Multiplying both sides of (11) by $t^{2 n}$ and summing for $n \geq 1$, we see that

$$
\sum_{n \geq 1} \frac{\bar{D}_{2 n+1}^{(1,0, \emptyset, 0)}(x) t^{2 n}}{(2 n) !}-x=x\left(\sum_{k \geq 0} \frac{\bar{D}_{2 n+1}^{(1,0, \emptyset, 0)}(x) t^{2 k}}{(2 k) !}\right)\left(\sum_{k \geq 0} \frac{B_{2 k+1}^{(1,0, \emptyset, 0)}(1) t^{2 k+1}}{(2 k+1) !}\right) .
$$

By (2),

$$
\sum_{k \geq 1} \frac{B_{2 k-1}(1) t^{2 k-1}}{(2 k-1) !}=\tan (t)
$$

so that

$$
\frac{\partial}{\partial t} \bar{D}^{(1,0, \emptyset, 0)}(t, x)=x+x \tan (x) \bar{D}^{(1,0, \emptyset, 0)}(t, x) .
$$

Our initial condition is that $\bar{D}^{(1,0, \emptyset, 0)}(0, x)=0$. One can easily check that the solution to this differential equation is

$$
\bar{D}^{(1,0, \emptyset, 0)}(t, x)=x(\sec (t))^{x} \int_{0}^{t}(\cos (z))^{x} d z .
$$

As observed above, the difference between $D_{2 n+1}^{(1,0, \emptyset, 0)}(x)$ and $\bar{D}_{2 n+1}^{(1,0, \emptyset, 0)}(x)$ is that the permutations $\sigma=\sigma_{1} \cdots \sigma_{2 n+1} \in D U_{2 n+1}$ such that $\sigma_{1}=2 n+1$ are weighted differently in that such permutations are weighted with an extra power of $x$ in $\bar{D}_{2 n+1}^{(1,0, \emptyset, 0)}(x)$ than they are in $D_{2 n+1}^{(1,0, \emptyset, 0)}(x)$. That is,

$$
x \sum_{\sigma \in D U_{2 n+1}, \sigma_{1}=2 n+1} x^{\mathrm{mmp}^{(1,0, \emptyset, 0)}(\sigma)}=\sum_{\sigma \in D U_{2 n+1, \sigma_{1}=2 n+1}} x^{\mathrm{mmp}^{(1,0, \emptyset, 0)}(\sigma)} x^{\chi\left(\sigma_{1}=2 n+1\right)} .
$$

It is easy to see that

$$
\sum_{\sigma \in D U_{2 n+1}, \sigma_{1}=2 n+1} x^{\mathrm{mmp}^{(1,0, \emptyset, 0)}(\sigma)}=A_{2 n}^{(1,0, \emptyset, 0)}(x) .
$$

Thus, it follows that

$$
D_{2 n+1}^{(1,0, \emptyset, 0)}(x)=\bar{D}_{2 n+1}^{(1,0, \emptyset, 0)}(x)+(1-x) A_{2 n}^{(1,0, \emptyset, 0)}(x) .
$$

Multiplying both sides of (13) by $\frac{t^{2 n+1}}{(2 n+1) !}$ and summing for $n \geq 0$, we see that

$$
D^{(1,0, \emptyset, 0)}(t, x)=\bar{D}^{(1,0, \emptyset, 0)}(t, x)+(1-x) \int_{0}^{t} A^{(1,0, \emptyset, 0)}(z, x) d z
$$




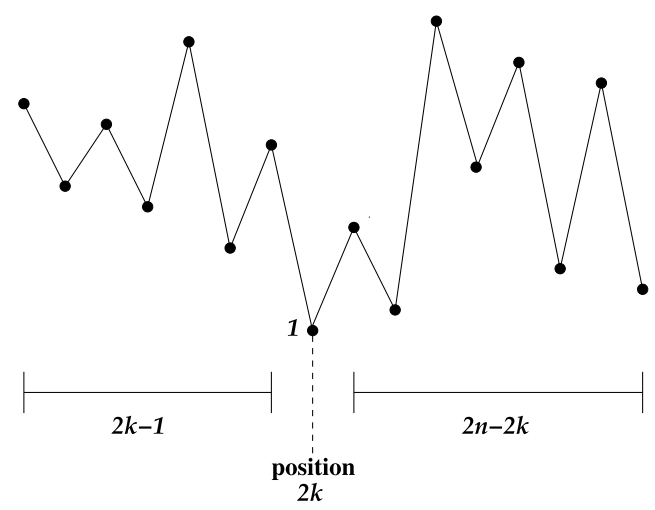

Figure 5: The graph of a $\sigma \in D U_{2 n}^{(2 k)}$.

Hence,

$$
D^{(1,0, \emptyset, 0)}(t, x)=x(\sec (t))^{x} \int_{0}^{t}(\cos (z))^{x} d z+(1-x) \int_{0}^{t}(\sec (z))^{x} d z
$$

\subsection{The generating function $C^{(1,0, \emptyset, 0)}(t, x)$}

If $\sigma=\sigma_{1} \cdots \sigma_{2 n} \in D U_{2 n}$, then 1 must occur in one of the positions $2,4, \ldots$, $2 n$. Let $D U_{2 n}^{(2 k)}$ denote the set of permutations $\sigma \in D U_{2 n}$ such that $\sigma_{2 k}=1$. A schematic diagram of an element in $D U_{2 n}^{(2 k)}$ is pictured in Figure 5.

Let

$$
C_{2 n}^{(1,0, \emptyset, 0)}(x, y)=\sum_{\sigma \in D U_{2 n}} x^{\mathrm{mmp}^{(1,0, \emptyset, 0)}(\sigma)} y^{\chi\left(\sigma_{1}=2 n\right)}
$$

First, we want to study the polynomial $\bar{C}_{2 n}^{(1,0, \emptyset, 0)}(x)=C_{2 n+1}^{(1,0, \emptyset, 0)}(x, x)$. As was the case with $D_{2 n+1}^{(1,0, \emptyset, 0)}(x)$, if $\sigma=\sigma_{1} \cdots \sigma_{2 n} \in D U_{2 n}$ and $\sigma_{1}<2 n$, then $\sigma_{1}$ will automatically contribute to $\mathrm{mmp}^{(1,0, \emptyset, 0)}(\sigma)$. However, if $\sigma_{1}=2 n$, then $\sigma_{1}$ will not contribute to $\mathrm{mmp}^{(1,0, \emptyset, 0)}(\sigma)$. Thus, the difference between $\bar{C}_{2 n}^{(1,0, \emptyset, 0)}(x)$ and $C_{2 n}^{(1,0, \emptyset, 0)}(x)$ is that $\sigma_{1}$ always contributes a factor of $x$ to $x^{\mathrm{mmp}^{(1,0, \emptyset, 0)}(\sigma)} x^{\chi\left(\sigma_{1}=2 n\right)}$.

First, consider $\sigma=\sigma_{1} \cdots \sigma_{2 n} \in D U_{2 n}^{(2 n)}$. Since $\sigma_{2 n}=1$, it is easy to see that

$$
\sum_{\sigma \in D U_{2 n}^{(2 n)}} x^{\mathrm{mmp}^{(1,0, \emptyset, 0)}(\sigma)} x^{\chi\left(\sigma_{1}=2 n\right)}=\bar{D}_{2 n-1}^{(1,0, \emptyset, 0)}(x)
$$


Next, consider a $\sigma=\sigma_{1} \cdots \sigma_{2 n} \in D U_{2 n}^{(2 k)}$ where $1 \leq k<n$. Note that there are $\left(\begin{array}{l}2 n-1 \\ 2 k-1\end{array}\right)$ ways to pick the elements which occur to the right of position $2 k$ in such a $\sigma$ and there are $C_{2 n-2 k}(1)=A_{2 n-2 k}(1)$ ways to order them since the elements to the right of position $2 k$ form a down-up permutation of length $2 n-2 k$. For a $\sigma=\sigma_{1} \cdots \sigma_{2 n+1} \in D U_{2 n}^{(2 k)}$, none of the elements $\sigma_{i}$ for $i>2 k$ matches $M M P(1,0, \emptyset, 0)$ in $\sigma$ and $\sigma_{2 k}=1$ always matches $M M P(1,0, \emptyset, 0)$ in $\sigma$. Thus, the only other elements of $\sigma$ that can possibly contribute to $\mathrm{mmp}^{(1,0, \emptyset, 0)}(\sigma) x^{\chi\left(\sigma_{1}=2 n\right)}$ are the elements $\sigma_{1}, \sigma_{2}, \sigma_{4}, \ldots, \sigma_{2 k-2}$. Since in $\bar{C}_{2 n+1}^{(1,0, \emptyset, 0)}(x), \sigma_{1}$ always contributes to $\mathrm{mmp}^{(1,0, \emptyset, 0)}(\sigma) x^{\chi\left(\sigma_{1}=2 n\right)}$ and the elements to the right of position $2 k$ have no effect on whether $\sigma_{2}, \ldots$, $\sigma_{2 k-2}$ contribute to $\mathrm{mmp}^{(1,0, \emptyset, 0)}(\sigma)$, it follows that the contribution of the elements to the left of position $2 k$ to $\sum_{\sigma \in D U_{2 n}^{(2 k)}} x^{\mathrm{mmp}^{(1,0, \emptyset, 0)}(\sigma)} x^{\chi\left(\sigma_{1}=2 n\right)}$ is $\bar{D}_{2 k-1}(x)$. Hence, for $n \geq 1$,

$$
\bar{C}_{2 n}^{(1,0, \emptyset, 0)}(x)=\bar{D}_{2 n-1}^{(1,0, \emptyset, 0)}(x)+x \sum_{k=1}^{n-1}\left(\begin{array}{l}
2 n-1 \\
2 k-1
\end{array}\right) \bar{D}_{2 k-1}^{(1,0, \emptyset, 0)}(x) A_{2 n-2 k}(1)
$$

or, equivalently,

$$
\frac{\bar{C}_{2 n}^{(1,0, \emptyset, 0)}(x)}{(2 n-1) !}=\frac{\bar{D}_{2 n-1}^{(1,0, \emptyset, 0)}(x)}{(2 n-1) !}+x \sum_{k=1}^{n-1} \frac{\bar{D}_{2 k-1}^{(1,0, \emptyset, 0)}(x)}{(2 k-1) !} \frac{A_{2 n-2 k}(1)}{(2 n-2 k) !} .
$$

Multiplying both sides of (14) by $t^{2 n-1}$ and summing for $n \geq 1$, we see that

$$
\begin{aligned}
\sum_{n \geq 1} \frac{\bar{C}_{2 n}^{(1,0, \emptyset, 0)}(x) t^{2 n-1}}{(2 n-1) !}= & \sum_{n \geq 1} \frac{\bar{D}_{2 n-1}^{(1,0, \emptyset, 0)}(x) t^{2 n-1}}{(2 n-1) !} \\
& +x\left(\sum_{k \geq 0} \frac{\bar{D}_{2 n+1}^{(1,0, \emptyset, 0)}(x) t^{2 n+1}}{(2 n+1) !}\right)\left(\sum_{k \geq 1} \frac{A_{2 k}(1) t^{2 k}}{(2 k) !}\right)
\end{aligned}
$$

By (1),

$$
\sum_{k \geq 0} \frac{A_{2 k}(1) t^{2 k}}{(2 k) !}=\sec (t)
$$


so that

$$
\begin{aligned}
\frac{\partial}{\partial t} \bar{C}^{(1,0, \emptyset, 0)}(t, x) & =\bar{D}^{(1,0, \emptyset, 0)}(t, x)+x \bar{D}^{(1,0, \emptyset, 0)}(t, x)(\sec (t)-1) \\
& =\bar{D}^{(1,0, \emptyset, 0)}(t, x)(1-x+x \sec (t)) \\
& =x(\sec (t))^{x}(1-x+x \sec (t)) \int_{0}^{t}(\cos (z))^{x} d z
\end{aligned}
$$

Our initial condition is that $\bar{C}^{(1,0, \emptyset, 0)}(0, x)=1$. Maple will give a solution to this differential equation, but it is a complicated expression which is not particularly useful for our purposes so that we will simply record the solution to this differential equation as

$$
\bar{C}^{(1,0, \emptyset, 0)}(t, x)=1+\int_{0}^{t}\left[x(\sec (z))^{x}(1-x+x \sec (z)) \int_{0}^{z}(\cos (y))^{x} d y\right] d z
$$

As observed above, the difference between $C_{2 n}^{(1,0, \emptyset, 0)}(x)$ and $\bar{C}_{2 n}^{(1,0, \emptyset, 0)}(x)$ is that the permutations $\sigma=\sigma_{1} \cdots \sigma_{2 n+1} \in D U_{2 n}$ such that $\sigma_{1}=2 n$ are weighted differently in that such permutations are weighted with an extra power of $x$ in $\bar{C}_{2 n}^{(1,0, \emptyset, 0)}(x)$ than they are in $C_{2 n}^{(1,0, \emptyset, 0)}(x)$. That is,

$$
x \sum_{\sigma \in D U_{2 n}, \sigma_{1}=2 n} x^{\mathrm{mmp}^{(1,0, \emptyset, 0)}(\sigma)}=\sum_{\sigma \in D U_{2 n}, \sigma_{1}=2 n} x^{\mathrm{mmp}^{(1,0, \emptyset, 0)}(\sigma)} x^{\chi\left(\sigma_{1}=2 n\right)} .
$$

It is easy to see that

$$
\sum_{\sigma \in D U_{2 n}, \sigma_{1}=2 n} x^{\mathrm{mmp}^{(1,0, \emptyset, 0)}(\sigma)}=B_{2 n-1}^{(1,0, \emptyset, 0)}(x)
$$

Thus, it follows that

$$
C_{2 n}^{(1,0, \emptyset, 0)}(x)=\bar{C}_{2 n}^{(1,0, \emptyset, 0)}(x)+(1-x) B_{2 n-1}^{(1,0, \emptyset, 0)}(x)
$$

Multiplying both sides of (16) by $\frac{t^{2 n}}{(2 n) !}$ and summing for $n \geq 0$, we see that

$$
C^{(1,0, \emptyset, 0)}(t, x)=\bar{C}^{(1,0, \emptyset, 0)}(t, x)+(1-x) \int_{0}^{t} B^{(1,0, \emptyset, 0)}(z, x) d z .
$$


Hence,

$$
\begin{aligned}
C^{(1,0, \emptyset, 0)}(t, x)= & 1+\int_{0}^{t}\left[x(\sec (z))^{x}(1-x+x \sec (z)) \int_{0}^{z}(\cos (y))^{x} d y\right] d z \\
& +(1-x) \int_{0}^{t} B^{(1,0, \emptyset, 0)}(z, x) d z .
\end{aligned}
$$

3. The coefficients of the polynomials $A_{2 n}^{(1,0, \emptyset, 0)}(x)$,

$$
B_{2 n+1}^{(1,0, \emptyset, 0)}(x), C_{2 n}^{(1,0, \emptyset, 0)}(x) \text {, and } D_{2 n+1}^{(1,0, \emptyset, 0)}(x) \text {. }
$$

The main goal of this section is to explain several of the coefficients of the polynomials $A_{2 n}^{(1,0, \emptyset, 0)}(x), B_{2 n+1}^{(1,0, \emptyset, 0)}(x), C_{2 n}^{(1,0, \emptyset, 0)}(x)$, and $D_{2 n+1}^{(1,0, \emptyset, 0)}(x)$. For $n \geq 1$, let $(2 n) ! !=\prod_{i=1}^{n} 2 i$ and $(2 n-1) ! !=\prod_{i=1}^{n}(2 i-1)$. First, it is easy to understand the coefficients of the lowest power of $x$ in each of these polynomials. That is, we have the following theorem.

\section{Theorem 3.}

(1) For all $n \geq 1$,

$$
\left.A_{2 n}^{(1,0, \emptyset, 0)}(x)\right|_{x}=B_{2 n-1}(1)
$$

(2) For all $n \geq 1$,

$$
\left.B_{2 n+1}^{(1,0, \emptyset, 0)}(x)\right|_{x}=A_{2 n}(1)+B_{2 n-1}(1)
$$

(3) For all $n \geq 2$,

$$
\left.C_{2 n}^{(1,0, \emptyset, 0)}(x)\right|_{x}=A_{2 n-2}(1)+B_{2 n-3}(1) .
$$

(4) For all $n \geq 1$,

$$
\left.D_{2 n+1}^{(1,0, \emptyset, 0)}(x)\right|_{x}=B_{2 n-1}(1) .
$$

Proof. For (1), note that if $\sigma=\sigma_{1} \cdots \sigma_{2 n} \in U D_{2 n}$ where $n \geq 1$, then $\sigma_{1}$ always matches $\operatorname{MMP}(1,0, \emptyset, 0)$ in $\sigma$. Moreover if $\sigma_{1} \neq 1$, then $\sigma_{2 k+1}=1$ for some $k \geq 1$ in which case $\sigma_{2 k+1}$ will also match $M M P(1,0, \emptyset, 0)$ in $\sigma$. Thus, the only possible way to have $\mathrm{mmp}^{(1,0, \emptyset, 0)}(\sigma)=1$ is if $\sigma_{1}=1$ in which case none of $\sigma_{2}, \ldots, \sigma_{2 n}$ will match $M M P(1,0, \emptyset, 0)$ in $\sigma$. Clearly, in such a situation, $\operatorname{red}\left(\sigma_{2} \cdots \sigma_{2 n}\right) \in D U_{2 n-1}$ so that we have $D_{2 n-1}(1)=B_{2 n-1}(1)$ ways to choose $\sigma_{2} \cdots \sigma_{2 n}$. It follows that $\left.A_{2 n}^{(1,0, \emptyset, 0)}(x)\right|_{x}=B_{2 n-1}(1)$ for $n \geq 1$.

For (2), note that if $\sigma=\sigma_{1} \cdots \sigma_{2 n+1} \in U D_{2 n+1}$ where $n \geq 1$, then again $\sigma_{1}$ always matches $M M P(1,0, \emptyset, 0)$ in $\sigma$. However, in this case, if 
$\sigma_{1} \neq 1$, then $\sigma_{2 k+1}=1$ for some $k \geq 1$ in which case $\sigma_{2 k+1}$ will also match $M M P(1,0, \emptyset, 0)$ in $\sigma$ if $1 \leq k<n$, but it will not match $\operatorname{MMP}(1,0, \emptyset, 0)$ in $\sigma$ if $k=n$. Thus, if $\sigma=\sigma_{1} \cdots \sigma_{2 n+1} \in U D_{2 n+1}$ and $\operatorname{mmp}^{(1,0, \emptyset, 0)}(\sigma)=$ 1 , it must be the case that $\sigma_{1}=1$ or $\sigma_{2 n+1}=1$. Now if $\sigma_{1}=1$, then none of $\sigma_{2}, \ldots, \sigma_{2 n+1}$ will match $M M P(1,0, \emptyset, 0)$ in $\sigma$. Clearly, in such a situation, $\operatorname{red}\left(\sigma_{2} \cdots \sigma_{2 n+1}\right) \in D U_{2 n}$ so that we have $C_{2 n}(1)=A_{2 n}(1)$ ways to choose $\sigma_{2} \cdots \sigma_{2 n+1}$. If $\sigma_{2 n+1}=1$, then it must be the case that $\sigma_{1}=2$ since otherwise $\sigma_{2 k+1}=2$ for some $k \geq 1$ in which case $\sigma_{1}$ and $\sigma_{2 k+1}=2$ will match $\operatorname{MMP}(1,0, \emptyset, 0)$ in $\sigma$. But then $\operatorname{red}\left(\sigma_{2} \cdots \sigma_{2 n}\right) \in D U_{2 n-1}$ so that we have $D_{2 n-1}(1)=B_{2 n-1}(1)$ ways to choose $\sigma_{2} \cdots \sigma_{2 n}$. Hence, $\left.B_{2 n+1}^{(1,0, \emptyset, 0)}(x)\right|_{x}=$ $A_{2 n}(1)+B_{2 n-1}(1)$ for $n \geq 1$.

For (3), note that if $\sigma=\sigma_{1} \cdots \sigma_{2 n} \in D U_{2 n}$ where $n \geq 2$, then $\sigma_{2}$ always matches $\operatorname{MMP}(1,0, \emptyset, 0)$ in $\sigma$. Moreover, if $\sigma_{2} \neq 1$, then $\sigma_{2 k}=1$ for some $k \geq 2$ in which case $\sigma_{2 k}$ will also match $M M P(1,0, \emptyset, 0)$ in $\sigma$ for $1<k<n$ but will not match $M M P(1,0, \emptyset, 0)$ in $\sigma$ if $k=n$. In addition, $\sigma_{1}$ will match $\operatorname{MMP}(1,0, \emptyset, 0)$ in $\sigma$ unless $\sigma_{1}=2 n$. Thus, if $\sigma=\sigma_{1} \cdots \sigma_{2 n} \in D U_{2 n}$ and $\operatorname{mmp}^{(1,0, \emptyset, 0)}(\sigma)=1$, then we must have $\sigma_{1}=2 n$ and either $\sigma_{2}=1$ or $\sigma_{2 n}=1$. If $\sigma_{2}=1$, then none of $\sigma_{3}, \ldots, \sigma_{2 n}$ will match $M M P(1,0, \emptyset, 0)$ in $\sigma$. Clearly, in such a situation, $\operatorname{red}\left(\sigma_{3} \cdots \sigma_{2 n}\right) \in D U_{2 n-2}$ so that we have $C_{2 n-2}(1)=A_{2 n-2}(1)$ ways to choose $\sigma_{3} \cdots \sigma_{2 n}$. If $\sigma_{2 n}=1$, then it must be the case that $\sigma_{2}=2$ since otherwise $\sigma_{2 k}=2$ for some $k \geq 2$ in which case $\sigma_{2}$ and $\sigma_{2 k}=2$ will match $M M P(1,0, \emptyset, 0)$ in $\sigma$. But then $\operatorname{red}\left(\sigma_{3} \cdots \sigma_{2 n-1}\right) \in$ $D U_{2 n-3}$ so that we have $D_{2 n-3}(1)=B_{2 n-3}(1)$ ways to choose $\sigma_{3} \cdots \sigma_{2 n-1}$. Hence, $\left.C_{2 n}^{(1,0, \emptyset, 0)}(x)\right|_{x}=A_{2 n-2}(1)+B_{2 n-3}(1)$ for $n \geq 2$.

For $(4)$, note that if $\sigma=\sigma_{1} \cdots \sigma_{2 n+1} \in D U_{2 n+1}$ where $n \geq 1$, then $\sigma_{2}$ always matches $\operatorname{MMP}(1,0, \emptyset, 0)$ in $\sigma$. Moreover if $\sigma_{2} \neq 1$, then $\sigma_{2 k}=1$ for some $k \geq 2$ in which case $\sigma_{2 k}$ will also match $\operatorname{MMP}(1,0, \emptyset, 0)$ in $\sigma$. Finally, $\sigma_{1}$ will also match $\operatorname{MMP}(1,0, \emptyset, 0)$ in $\sigma$ unless $\sigma_{1}=2 n+1$. Thus, if $\sigma=\sigma_{1} \cdots \sigma_{2 n+1} \in D U_{2 n+1}$ and $\operatorname{mmp}^{(1,0, \emptyset, 0)}(\sigma)=1$, it must be the case that $\sigma_{1}=2 n+1$ and $\sigma_{2}=1$ in which case none of $\sigma_{3}, \cdots, \sigma_{2 n+1}$ will match $M M P(1,0, \emptyset, 0)$ in $\sigma$. Clearly, in such a situation, $\operatorname{red}\left(\sigma_{3} \cdots \sigma_{2 n}\right) \in D U_{2 n-1}$ so that we have $D_{2 n-1}(1)=B_{2 n-1}(1)$ ways to choose $\sigma_{3} \cdots \sigma_{2 n+1}$. It follows that $\left.D_{2 n+1}^{(1,0, \emptyset, 0)}(x)\right|_{x}=B_{2 n-1}(1)$ for $n \geq 1$.

We can also explain the coefficients of the highest power of $x$ in each of the polynomials $A_{2 n}(x), B_{2 n+1}(x)$, and $D_{2 n+1}(x)$. That is, we have the following theorem.

\section{Theorem 4.}

(1) For all $n \geq 1$, the highest power of $x$ that appears in $A_{2 n}^{(1,0, \emptyset, 0)}(x)$ is $x^{n}$ which appears with coefficient $(2 n-1)$ !!. 
(2) For all $n \geq 1$, the highest power of $x$ that appears in $B_{2 n+1}^{(1,0, \emptyset, 0)}(x)$ is $x^{n}$ which appears with coefficient $(n+1)((2 n-1) ! !)$.

(3) For all $n \geq 2$, the highest power of $x$ that appears in $C_{2 n}^{(1,0, \emptyset, 0)}(x)$ is $x^{n}$ which appears with coefficient $\left(2 n^{2}-n-1\right)((2 n-4) ! !)-n((2 n-3) ! !)$.

(4) For all $n \geq 2$, the highest power of $x$ that appears in $D_{2 n+1}^{(1,0, \emptyset, 0)}(x)$ is $x^{n+1}$ which appears with coefficient $(2 n) ! !-(2 n-1) ! !$.

Proof. For (1), we proceed by induction on $n$. Clearly, the formula holds for $n=1$ since $A_{2}^{(1,0, \emptyset, 0)}(x)=x$. Thus, assume that $n>1$, and that by induction, we know that $\left.A_{2 n-2}^{(1,0, \emptyset, 0)}(x)\right|_{x^{n-1}}=(2 n-3)$ !!. It is easy to see that the maximum that $\operatorname{mmp}^{(1,0, \emptyset, 0)}(\sigma)$ can be is $n$ since for any $\sigma=\sigma_{1} \cdots \sigma_{2 n} \in$ $U D_{2 n}$ only $\sigma_{1}, \sigma_{3}, \cdots, \sigma_{2 n-1}$ can match $M M P(1,0, \emptyset, 0)$ in $\sigma$. If $\sigma_{2 k+1}=1$ for some $k<n-1$, then $\sigma_{2 k+3}, \ldots, \sigma_{2 n-1}$ will not match $\operatorname{MMP}(1,0, \emptyset, 0)$ in $\sigma$. Thus, if $\sigma=\sigma_{1} \cdots \sigma_{2 n} \in U D_{2 n}$ is such that $\operatorname{mmp}^{(1,0, \emptyset, 0)}(\sigma)=n$, then $\sigma_{2 n-1}=1$ and $\operatorname{mmp}^{(1,0, \emptyset, 0)}\left(\sigma_{1} \cdots \sigma_{2 n-2}\right)=n-1$. We then have $(2 n-1)$ ways to choose the value $\sigma_{2 n}$, and once we have chosen the value of $\sigma_{2 n}$, we have $(2 n-3)$ !! ways to choose $\sigma_{1} \cdots \sigma_{2 n-2}$. Hence, $\left.A^{(1,0, \emptyset, 0)}(x)\right|_{x^{n}}=(2 n-1) !$ !.

For (2), it is easy to see that our formula holds for $n=1$ and $n=2$ since $\left.B_{3}^{(1,0, \emptyset, 0)}(x)\right|_{x}=2$ and $\left.B_{5}^{(1,0, \emptyset, 0)}(x)\right|_{x^{2}}=9=3(3 ! !)$. So assume that $n \geq 3$ and suppose that $\sigma=\sigma_{1} \cdots \sigma_{2 n+1} \in U D_{2 n+1}$. Then only $\sigma_{1}, \sigma_{3}, \ldots, \sigma_{2 n-1}$ can match the $\operatorname{MMP}(1,0, \emptyset, 0)$ in $\sigma$. Thus, the maximum that $\operatorname{mmp}^{(1,0, \emptyset, 0)}(\sigma)$ can be is $n$. Note that if $\sigma_{2 k+1}=1$ where $0 \leq k<n-1$, then none of $\sigma_{2 j+1}$ for $j>k$ will match the $\operatorname{MMP}(1,0, \emptyset, 0)$ in $\sigma$. It follows that if $\operatorname{mmp}^{(1,0, \emptyset, 0)}(\sigma)=$ $n$, then it must be the case that $\sigma_{2 n+1}=1$ or $\sigma_{2 n-1}=1$. Now if $\sigma_{2 n-1}=1$, then we have $\left(\begin{array}{c}2 n \\ 2\end{array}\right)$ ways to choose the values of $\sigma_{2 n}$ and $\sigma_{2 n+1}$, and it must be the case that $\operatorname{red}\left(\sigma_{1} \cdots \sigma_{2 n-2}\right)=\tau$ where $\tau \in U D_{2 n-2}$ and $\operatorname{mmp}^{(1,0, \emptyset, 0)}(\tau)=$ $n-1$. It then follows from part (1) that we have $(2 n-3)$ !! ways to choose $\sigma_{1} \cdots \sigma_{2 n-2}$ so that the set of permutations $\sigma=\sigma_{1} \cdots \sigma_{2 n+1} \in U D_{2 n+1}$ with $\sigma_{2 n-1}=1$ contributes $\left(\begin{array}{c}2 n \\ 2\end{array}\right)(2 n-3) ! !=n((2 n-1) ! !)$ to $\left.B_{2 n+1}^{(1,0, \emptyset, 0)}(x)\right|_{x^{n}}$. If $\sigma_{2 n+1}=1$, then it must be the case that $\sigma_{2 n-1}=2$ and $\operatorname{red}\left(\sigma_{1} \cdots \sigma_{2 n-2}\right)=\tau$ where $\tau \in U D_{2 n-2}$ and $\operatorname{mmp}^{(1,0, \emptyset, 0)}(\tau)=n-1$. Thus, we have $2 n-1$ choices for the value of $\sigma_{2 n}$, and then as before we have $(2 n-3)$ !! to choose $\sigma_{1} \cdots \sigma_{2 n-2}$. Thus, the set of permutations $\sigma=\sigma_{1} \cdots \sigma_{2 n+1} \in U D_{2 n+1}$ with $\sigma_{2 n+1}=1$ contributes $(2 n-1)$ !! to $\left.B_{2 n+1}^{(1,0, \emptyset, 0)}(x)\right|_{x^{n}}$. Hence, $\left.B_{2 n+1}^{(1,0, \emptyset, 0)}(x)\right|_{x^{n}}=$ $(n+1)(2 n-1) ! !$.

For (4), it is easy to see that if $\sigma=\sigma_{1} \cdots \sigma_{2 n+1} \in D U_{2 n+1}$, then only $\sigma_{1}, \sigma_{2}, \sigma_{4}, \ldots, \sigma_{2 n}$ can match $\operatorname{MMP}(1,0, \emptyset, 0)$ in $\sigma$. Thus, $\operatorname{mmp}^{(1,0, \emptyset, 0)}(\sigma)$ is at most $n+1$. It is also easy to see that if $\sigma_{2 k}=1$ for $k<n$, then $\sigma_{2 k+2}, \ldots, \sigma_{2 n}$ will not match $\operatorname{MMP}(1,0, \emptyset, 0)$ in $\sigma$ so that if $\operatorname{mmp}^{(1,0, \emptyset, 0)}(\sigma)=$ 
$n+1$, then it must be the case that $\sigma_{2 n}=1$. Thus, assume that $\sigma=$ $\sigma_{1} \cdots \sigma_{2 n+1} \in D U_{2 n+1}$ is such that $\mathrm{mmp}^{(1,0, \emptyset, 0)}(\sigma)=n+1$. Since $\sigma_{2 n}=1$, we have two cases.

Case 1. $\sigma_{2 n+1}=2 n+1$. In this case, we know that $\sigma_{1}$ will always match $M M P(1,0, \emptyset, 0)$ in $\sigma$. Thus, as far as $\sigma_{1} \cdots \sigma_{2 n-1}$ is concerned, we are dealing with the polynomial $\bar{D}_{2 n-1}^{(1,0, \emptyset, 0)}(x)$. We then have the following lemma.

Lemma 5. For $n \geq 1$, the highest power of $x$ which occurs in $\bar{D}_{2 n+1}^{(1,0, \emptyset, 0)}(x)$ is $x^{n+1}$ which occurs with a coefficient of $(2 n) ! !$.

Proof. We proceed by induction on $n$. Our theorem holds for $n=1$ since $\bar{D}_{3}^{(1,0, \emptyset, 0)}(x)=2 x^{2}$. Now assume that $n>1$ and the lemma holds for $n-1$. As in our discussion for $D_{2 n+1}(x)$, if $\sigma=\sigma_{1} \cdots \sigma_{2 n+1} \in D U_{2 n+1}$ is such that $\chi\left(\sigma_{1}=2 n+1\right)+\operatorname{mmp}^{(1,0, \emptyset, 0)}(\sigma)=n+1$, then it must be the case that $\sigma_{2 n}=1$. But then we have $2 n$ choices for $\sigma_{2 n+1}$, and once we have chosen $\sigma_{2 n+1}$, then $\tau=\operatorname{red}\left(\sigma_{1} \cdots \sigma_{2 n-1}\right)$ must be an element of $D U_{2 n-1}$ such that $\chi\left(\tau_{1}=2 n-1\right)+\mathrm{mmp}^{(1,0, \emptyset, 0)}(\tau)=n$. By induction, we have $(2(n-1)) !$ ways to pick $\sigma_{1} \cdots \sigma_{2 n-1}$. Thus, $\left.\bar{D}_{2 n+1}^{(1,0, \emptyset, 0)}(x)\right|_{x^{n}}=(2 n) ! !$.

It follows that in Case 1 , we have $(2 n-2)$ !! ways to pick $\sigma_{1} \cdots \sigma_{2 n-1}$ so that the permutations such that $\sigma_{2 n}=1$ and $\sigma_{2 n+1}=2 n+1$ contribute $(2 n-2)$ !! to $\left.D_{2 n+1}^{(1,0, \emptyset, 0)}(x)\right|_{x^{n+1}}$.

Case 2. $\sigma_{2 n+1}<2 n+1$. In this case, $\tau=\operatorname{red}\left(\sigma_{1} \cdots \sigma_{2 n-1}\right)$ must be an element of $D U_{2 n-1}$ such that $\mathrm{mmp}^{(1,0, \emptyset, 0)}(\tau)=n$. It then follows by induction that we have $(2 n-1)$ ways to pick $\sigma_{2 n+1}$, and once we have chosen $\sigma_{2 n+1}$, we have $(2 n-2) ! !-(2 n-3)$ !! ways to pick $\sigma_{1} \cdots \sigma_{2 n-1}$. Hence, the permutations such that $\sigma_{2 n}=1$ and $\sigma_{2 n+1}<2 n+1$ contribute $(2 n-1)((2 n-2) ! !-(2 n-3) ! !)=(2 n-1)((2 n-2) ! !)-(2 n-1) ! !$ to $\left.D_{2 n+1}^{(1,0, \emptyset, 0)}(x)\right|_{x^{n+1}}$.

Thus,

$$
\begin{aligned}
\left.D_{2 n+1}^{(1,0, \emptyset, 0)}(x)\right|_{x^{n+1}} & =(2 n-2) ! !+(2 n-1)((2 n-2) ! !)-(2 n-1) ! ! \\
& =(2 n) ! !-(2 n-1) ! !
\end{aligned}
$$

For (3), observe that if $\sigma=\sigma_{1} \cdots \sigma_{2 n} \in D U_{2 n}$, then only $\sigma_{1}, \sigma_{2}, \sigma_{4}, \ldots$, $\sigma_{2 n-2}$ can match $\operatorname{MMP}(1,0, \emptyset, 0)$ so that $\mathrm{mmp}^{(1,0, \emptyset, 0)}(\sigma)$ is at most $n$. It is also easy to see that if $\sigma_{2 k}=1$ for $k<n-1$, then $\sigma_{2 k+2}, \ldots, \sigma_{2 n-2}$ will not match $M M P(1,0, \emptyset, 0)$ in $\sigma$ so that if $\mathrm{mmp}^{(1,0, \emptyset, 0)}(\sigma)=n$, then it must be the case that $\sigma_{2 n}=1$ or $\sigma_{2 n-2}=1$. Suppose that $\sigma=\sigma_{1} \cdots \sigma_{2 n} \in D U_{2 n}$ and $\mathrm{mmp}^{(1,0, \emptyset, 0)}(\sigma)=n$. We then have three cases. 
Case I. $\sigma_{2 n}=1$. In this case, it must be that $\tau=\operatorname{red}\left(\sigma_{1} \cdots \sigma_{2 n-1}\right) \in$ $D U_{2 n-1}$ and $\mathrm{mmp}^{(1,0, \emptyset, 0)}(\tau)=n$. Thus, by part $(3)$, we have $(2 n-2) ! !$ $(2 n-3) !$ ! choices for $\sigma_{1} \cdots \sigma_{2 n}$.

Case II. $\sigma_{2 n-2}=1$ and $\sigma_{2 n-1}=2 n$. In this case, we have $(2 n-2)$ choices for $\sigma_{2 n}$. The fact that $\sigma_{2 n-1}=2 n$ implies that $\sigma_{1}$ will always match $M M P(1,0$, $\emptyset, 0)$ so that $\gamma=\operatorname{red}\left(\sigma_{1} \cdots \sigma_{2 n-3}\right)$ is a permutation in $D U_{2 n-3}$ such that $\chi\left(\sigma_{1}=2 n-3\right)+\mathrm{mmp}^{(1,0, \emptyset, 0)}(\gamma)=n-1$. By Lemma 5 , we will have $(2 n-4) ! !$ choices for $\sigma_{1} \cdots \sigma_{2 n-3}$ once we have chosen $\sigma_{2 n}$. Thus, the permutations in Case II will contribute $(2 n-2)$ !! to $\left.C_{2 n}^{(1,0, \emptyset, 0)}(x)\right|_{x^{n}}$.

Case III. $\sigma_{2 n-2}=1$ and $\sigma_{2 n-1}<2 n$. In this case, $\tau=\operatorname{red}\left(\sigma_{1} \cdots \sigma_{2 n-3}\right)$ must be an element of $D U_{2 n-3}$ such that $\mathrm{mmp}^{(1,0, \emptyset, 0)}(\tau)=n-1$. Then we have $\left(\begin{array}{c}2 n-2 \\ 2\end{array}\right)$ ways to pick $\sigma_{2 n-1}$ and $\sigma_{2 n}$, and once we have chosen $\sigma_{2 n-1}$ and $\sigma_{2 n}$, we have $(2 n-4) ! !-(2 n-5) ! !$ ways to pick $\sigma_{1} \cdots \sigma_{2 n-3}$ by part (4). It follows that the permutations in Case III contribute $\left(\begin{array}{c}2 n-2 \\ 2\end{array}\right)((2 n-4) ! !-$ $(2 n-5) ! !)$ to $\left.C_{2 n}^{(1,0, \emptyset, 0)}(x)\right|_{x^{n}}$.

Thus,

$$
\begin{aligned}
\left.C_{2 n}^{(1,0, \emptyset, 0)}(x)\right|_{x^{n}}= & (2 n-2) ! !-(2 n-3) ! !+(2 n-2) ! ! \\
& +\left(\begin{array}{c}
2 n-2 \\
2
\end{array}\right)((2 n-4) ! !-(2 n-5) ! !) \\
= & 2((2 n-2) ! !)-(2 n-3) ! ! \\
& +(n-1)(2 n-3)((2 n-4) ! !-(2 n-5) ! !) \\
= & (2(2 n-2)+(n-1)(2 n-3))((2 n-4) ! !)-n((2 n-3) ! !) \\
= & \left(2 n^{2}-n-1\right)((2 n-4) ! !)-n((2 n-3) ! !) .
\end{aligned}
$$

Next, we give formulas for the coefficient of $x^{2}$ in the polynomials $A_{2 n}^{(1,0, \emptyset, 0)}(x), B_{2 n+1}^{(1,0, \emptyset, 0)}(x), C_{2 n}^{(1,0, \emptyset, 0)}(x)$, and $B_{2 n+1}^{(1,0, \emptyset, 0)}(x)$. None of the corresponding sequences had previously appeared in the OEIS [12].

\section{Theorem 6.}

(1) For $n \geq 2$,

$$
\left.A_{2 n}^{(1,0, \emptyset, 0)}(x)\right|_{x^{2}}=\sum_{k=1}^{n-1}\left(\begin{array}{c}
2 n-1 \\
2 k
\end{array}\right) B_{2 k-1}(1) B_{2 n-2 k-1}(1) .
$$


(2) For $n \geq 3$,

$$
\left.B_{2 n+1}^{(1,0, \emptyset, 0)}(x)\right|_{x^{2}}=\left.A_{2 n}^{(1,0, \emptyset, 0)}(x)\right|_{x^{2}}+\sum_{k=1}^{n-1}\left(\begin{array}{l}
2 n \\
2 k
\end{array}\right) B_{2 k-1}(1) A_{2 n-2 k}(1) .
$$

(3) For $n \geq 2$,

$$
\left.D_{2 n+1}^{(1,0, \emptyset, 0)}(x)\right|_{x^{2}}=(2 n-1) B_{2 n-1}(1)+\sum_{k=2}^{n-1}\left(\begin{array}{l}
2 n-1 \\
2 k-2
\end{array}\right) B_{2 k-3}(1) B_{2 n-2 k+1}(1) .
$$

(4) For $n \geq 2$,

$$
\begin{aligned}
\left.C_{2 n}^{(1,0, \emptyset, 0)}(x)\right|_{x^{2}}= & \left.D_{2 n-1}^{(1,0, \emptyset, 0)}(x)\right|_{x^{2}}+(2 n-2) A_{2 n-2}(1) \\
& +\sum_{k=2}^{n-1}\left(\begin{array}{l}
2 n-2 \\
2 k-2
\end{array}\right) B_{2 k-3}(1) A_{2 n-2 k}(1) .
\end{aligned}
$$

Proof. For (1), suppose that $\sigma=\sigma_{1} \cdots \sigma_{2 n} \in U D_{2 n}$ and $\mathrm{mmp}^{(1,0, \emptyset, 0)}(\sigma)=2$. Then it cannot be that $\sigma_{1}=1$ since that would force that $\sigma_{2}, \ldots, \sigma_{2 n}$ do not match $\operatorname{MMP}(1,0, \emptyset, 0)$ in $\sigma$. Thus, $1 \in\left\{\sigma_{2 k+1}: k=1, \ldots, n-1\right\}$. Now suppose that $\sigma_{2 k+1}=1$ where $1 \leq k \leq n-1$. Then $\sigma_{2 k+1}$ will match $M M P(1,0, \emptyset, 0)$ in $\sigma$ and $\sigma_{2 k+2}, \ldots, \sigma_{2 n}$ will not match $M M P(1,0, \emptyset, 0)$ in $\sigma$. Hence, it must be the case that $\tau=\operatorname{red}\left(\sigma_{1} \cdots \sigma_{2 k}\right)$ is a permutation in $U D_{2 k}$ such that $\mathrm{mmp}^{(1,0, \emptyset, 0)}(\tau)=1$. Thus, we have $\left(\begin{array}{c}2 n-1 \\ 2 k\end{array}\right)$ ways to choose the set of elements for $\sigma_{1}, \ldots, \sigma_{2 k}$, and by Theorem 3 , we have $B_{2 k-1}(1)$ ways to order them. We also have $B_{2 n-2 k-1}(1)$ ways to order $\sigma_{2 k+2} \cdots \sigma_{2 n}$. Hence,

$$
\left.A_{2 n}^{(1,0, \emptyset, 0)}(x)\right|_{x^{2}}=\sum_{k=1}^{n-1}\left(\begin{array}{c}
2 n-1 \\
2 k
\end{array}\right) B_{2 k-1}(1) B_{2 n-2 k-1}(1) .
$$

The argument for (2) is similar. That is, suppose $\sigma=\sigma_{1} \cdots \sigma_{2 n+1} \in$ $U D_{2 n+1}$ and $\mathrm{mmp}^{(1,0, \emptyset, 0)}(\sigma)=2$. Then again we cannot have $\sigma_{1}=1$. Thus, $1 \in\left\{\sigma_{2 k+1}: k=1, \ldots, n\right\}$. Now suppose $\sigma_{2 k+1}=1$ where $1 \leq k \leq$ $n-1$. Then $\sigma_{2 k+1}$ will match $M M P(1,0, \emptyset, 0)$ in $\sigma$ and $\sigma_{2 k+2}, \ldots, \sigma_{2 n+1}$ will not match $M M P(1,0, \emptyset, 0)$ in $\sigma$. Hence, it must be the case that $\tau=$ $\operatorname{red}\left(\sigma_{1} \cdots \sigma_{2 k}\right)$ is a permutation in $U D_{2 k}$ such that $\mathrm{mmp}^{(1,0, \emptyset, 0)}(\tau)=1$. Thus, we have $\left(\begin{array}{c}2 n \\ 2 k\end{array}\right)$ ways to choose the set of elements for $\sigma_{1}, \ldots, \sigma_{2 k}$, and by Theorem 3, we have $B_{2 k-1}(1)$ ways to order them. We also have $A_{2 n-2 k}(1)$ ways to order $\sigma_{2 k+2} \cdots \sigma_{2 n+1}$. However if $\sigma_{2 n+1}=1$, then $\sigma_{2 n+1}$ does not match 
$\operatorname{MMP}(1,0, \emptyset, 0)$ in $\sigma$ so that it must be the case that $\alpha=\operatorname{red}\left(\sigma_{1} \cdots \sigma_{2 n}\right)$ is an element of $U D_{2 n}$ such that $\mathrm{mmp}^{(1,0, \emptyset, 0)}(\tau)=2$. It follows that in this case, we have $\left.A_{2 n}^{(1,0, \emptyset, 0)}(x)\right|_{x^{2}}$ ways to choose $\sigma_{1} \cdots \sigma_{2 n}$. Hence,

$$
\left.B_{2 n+1}^{(1,0, \emptyset, 0)}(x)\right|_{x^{2}}=\left.A_{2 n}^{(1,0, \emptyset, 0)}(x)\right|_{x^{2}}+\sum_{k=1}^{n-1}\left(\begin{array}{l}
2 n \\
2 k
\end{array}\right) B_{2 k-1}(1) A_{2 n-2 k}(1) .
$$

For part (3), suppose that $\sigma=\sigma_{1} \cdots \sigma_{2 n+1} \in D U_{2 n+1}$ and $\mathrm{mmp}^{(1,0, \emptyset, 0)}(\sigma)=2$. Then $1 \in\left\{\sigma_{2}, \sigma_{4}, \ldots, \sigma_{2 n}\right\}$. Now if $\sigma_{2}=1$, then we cannot have $\sigma_{1}=2 n+1$ because that would force $\mathrm{mmp}^{(1,0, \emptyset, 0)}(\sigma)=1$. Thus, if $\sigma_{2}=1$, then $2 \leq \sigma_{1} \leq 2 n$ in which case $\sigma_{1}$ and $\sigma_{2}$ will be the only two elements of $\sigma$ to match $M M P(1,0, \emptyset, 0)$ in $\sigma$. We then have $D_{2 n-1}(1)=$ $B_{2 n-1}(1)$ ways to pick $\sigma_{3} \cdots \sigma_{2 n+1}$ as $\operatorname{red}\left(\sigma_{3} \cdots \sigma_{2 n+1}\right) \in D U_{2 n-1}$. Thus, the number of $\sigma=\sigma_{1} \cdots \sigma_{2 n+1} \in D U_{2 n+1}$ such that $\mathrm{mmp}^{(1,0, \emptyset, 0)}(\sigma)=2$ and $\sigma_{2}=1$ is $(2 n-1) B_{2 n-1}(1)$. Next, assume that $\sigma_{2 k}=1$ where $2 \leq k \leq n$. Then $\sigma_{2 k}$ matches $\operatorname{MMP}(1,0, \emptyset, 0)$ in $\sigma$. It follows that we cannot have $2 n+1 \in\left\{\sigma_{3}, \ldots, \sigma_{2 n+1}\right\}$ since otherwise $\sigma_{1}$ and $\sigma_{2}$ would also match $\operatorname{MMP}(1,0, \emptyset, 0)$ in $\sigma$ which would force $\mathrm{mmp}^{(1,0, \emptyset, 0)}(\sigma) \geq 3$. Thus, it must be the case that $\sigma_{1}=2 n+1$. Moreover, if $s=\min \left(\left\{\sigma_{2}, \ldots, \sigma_{2 k-1}\right\}\right)$, then it must be the case that $\sigma_{2}=s$ since otherwise $s=\sigma_{2 j}$ for some $2 \leq j \leq k-1$ in which case both $\sigma_{2}$ and $\sigma_{2 j}$ would match $M M P(1,0, \emptyset, 0)$ in $\sigma$ which would mean $\mathrm{mmp}^{(1,0, \emptyset, 0)}(\sigma) \geq 3$. Thus, we have $\left(\begin{array}{l}2 n-1 \\ 2 k-2\end{array}\right)$ ways to choose the elements $\sigma_{3}, \ldots, \sigma_{2 k-1}$ and then we have $B_{2 k-3}(1)$ ways to order $\sigma_{3}, \ldots, \sigma_{2 k-1}$ since $\operatorname{red}\left(\sigma_{3} \cdots \sigma_{2 k-1}\right)$ must be an element of $D U_{2 k-3}$, and we have $B_{2 n-2 k+1}(1)$ ways to order $\sigma_{2 k+1} \cdots \sigma_{2 n+1}$ since $\operatorname{red}\left(\sigma_{2 k+1} \cdots \sigma_{2 n+1}\right)$ must be an element of $D U_{2 n-2 k+1}$. Hence,

$$
\left.D_{2 n+1}^{(1,0, \emptyset, 0)}(x)\right|_{x^{2}}=(2 n-1) B_{2 n-1}(1)+\sum_{k=2}^{n-1}\left(\begin{array}{c}
2 n-1 \\
2 k-2
\end{array}\right) B_{2 k-3}(1) B_{2 n-2 k+1}(1) .
$$

For part (4), suppose that $\sigma=\sigma_{1} \cdots \sigma_{2 n} \in D U_{2 n}$ and $\mathrm{mmp}^{(1,0, \emptyset, 0)}(\sigma)=$ 2. Then $1 \in\left\{\sigma_{2}, \sigma_{4}, \ldots, \sigma_{2 n}\right\}$. We then have three cases.

Case 1. $\sigma_{2 n}=1$. In this case, $\sigma_{2 n}$ does not match $M M P(1,0, \emptyset, 0)$ in $\sigma$ so that it must be the case that if $\tau=\operatorname{red}\left(\sigma_{1} \cdots \sigma_{2 n-1}\right)$, then $\tau$ is a permutation in $D U_{2 n-1}$ such that $\mathrm{mmp}^{(1,0, \emptyset, 0)}(\tau)=2$. Thus, , we have $\left.D_{2 n-1}^{(1,0, \emptyset, 0)}(x)\right|_{x^{2}}$ choices for $\sigma_{1} \cdots \sigma_{2 n-1}$.

Case 2. $\sigma_{2}=1$. In this case, we cannot have $\sigma_{1}=2 n$ because that would force $\mathrm{mmp}^{(1,0, \emptyset, 0)}(\sigma)=1$. Thus, if $\sigma_{2}=1$, then $2 \leq \sigma_{1} \leq 2 n-1$ 
in which case $\sigma_{1}$ and $\sigma_{2}$ will be the only two elements of $\sigma$ to match $M M P(1,0, \emptyset, 0)$ in $\sigma$. We then have $C_{2 n-2}(1)=A_{2 n-2}(1)$ ways to pick $\sigma_{3} \cdots \sigma_{2 n}$ as $\operatorname{red}\left(\sigma_{3} \cdots \sigma_{2 n}\right) \in D U_{2 n-2}$. Thus, the number of $\sigma=\sigma_{1} \cdots \sigma_{2 n} \in$ $D U_{2 n}$ such that $\mathrm{mmp}^{(1,0, \emptyset, 0)}(\sigma)=2$ and $\sigma_{2}=1$ is $(2 n-2) A_{2 n-2}(1)$.

Case 3. $\sigma_{2 k}=1$ where $2 \leq k \leq n-1$. Then $\sigma_{2 k}$ matches $M M P(1,0, \emptyset, 0)$ in $\sigma$. It follows that we cannot have $2 n+1 \in\left\{\sigma_{3}, \ldots, \sigma_{2 n}\right\}$ since otherwise $\sigma_{1}$ and $\sigma_{2}$ would also match $M M P(1,0, \emptyset, 0)$ in $\sigma$ which would force $\mathrm{mmp}^{(1,0, \emptyset, 0)}(\sigma) \geq 3$. Thus, it must be the case that $\sigma_{1}=2 n$. Again, if $s=\min \left(\left\{\sigma_{2}, \ldots, \sigma_{2 k-1}\right\}\right)$, then it must be the case that $\sigma_{2}=s$ since otherwise $s=\sigma_{2 j}$ for some $2 \leq j \leq k-1$ in which case both $\sigma_{2}$ and $\sigma_{2 j}$ would match $M M P(1,0, \emptyset, 0)$ in $\sigma$ which would force $\mathrm{mmp}^{(1,0, \emptyset, 0)}(\sigma) \geq 3$. It follows that we have $\left(\begin{array}{c}2 n-3 \\ 2 k-2\end{array}\right)$ ways to choose the elements $\sigma_{2}, \ldots, \sigma_{2 k-1}$ and then we have $B_{2 k-3}(1)$ ways to order $\sigma_{3}, \ldots, \sigma_{2 k-1} \operatorname{since} \operatorname{red}\left(\sigma_{3} \cdots \sigma_{2 k-1}\right)$ must be an element of $D U_{2 n-3}$ and $A_{2 n-2 k}(1)$ ways to order $\sigma_{2 k+1} \cdots \sigma_{2 n}$ since $\operatorname{red}\left(\sigma_{2 k+1} \cdots \sigma_{2 n}\right)$ must be an element of $D U_{2 n-2 k}$. Hence, the elements in Case 3 contribute $\sum_{k=2}^{n-1}\left(\begin{array}{c}2 n-3 \\ 2 k-2\end{array}\right) B_{2 k-3}(1) A_{2 n-2 k}(1)$ to $\left.C_{2 n}^{(1,0, \emptyset, 0)}(x)\right|_{x^{2}}$. Hence,

$$
\begin{aligned}
\left.C_{2 n}^{(1,0, \emptyset, 0)}(x)\right|_{x^{2}}= & \left.D_{2 n-1}^{(1,0, \emptyset, 0)}(x)\right|_{x^{2}}+(2 n-2) A_{2 n-2}(1) \\
& +\sum_{k=2}^{n-1}\left(\begin{array}{l}
2 n-2 \\
2 k-2
\end{array}\right) B_{2 k-3}(1) A_{2 n-2 k}(1) .
\end{aligned}
$$

Finally, we have the following theorem which gives formulas for the second highest coefficient in $A_{2 n}^{(1,0, \emptyset, 0)}(x), B_{2 n+1}^{(1,0, \emptyset, 0)}(x), C_{2 n}^{(1,0, \emptyset, 0)}(x)$, and $D_{2 n+1}^{(1,0, \emptyset, 0)}(x)$. None of the corresponding sequences had previously appeared in the OEIS [12].

Theorem 7. (1) For all $n \geq 2$,

$$
\left.A_{2 n}^{(1,0, \emptyset, 0)}(x)\right|_{x^{n-1}}=\frac{2}{3}\left(\begin{array}{l}
n \\
2
\end{array}\right)((2 n-1) ! !)
$$

(2) For all $n \geq 2$,

$$
\left.B_{2 n+1}^{(1,0, \emptyset, 0)}(x)\right|_{x^{n-1}}=\left(\frac{7}{3}\left(\begin{array}{l}
n \\
2
\end{array}\right)+2\left(\begin{array}{l}
n \\
3
\end{array}\right)\right)((2 n-1) ! !) .
$$


(3) For all $n \geq 1$,

$$
\begin{aligned}
\left.D_{2 n+1}^{(1,0, \emptyset, 0)}(x)\right|_{x^{n}}= & \left(\sum_{k=1}^{n} \frac{(5 k-4) k}{3}((2 k-2) ! !) \prod_{i=k+1}^{n}(2 i-1)\right) \\
& -\frac{2}{3}\left(\left(\begin{array}{l}
n \\
2
\end{array}\right)-1\right)((2 n-1) ! !) .
\end{aligned}
$$

(4) For all $n \geq 3$,

$$
\begin{aligned}
\left.C_{2 n}^{(1,0, \emptyset, 0)}(x)\right|_{x^{n-1}}= & \left.D_{2 n-1}^{(1,0, \emptyset, 0)}(x)\right|_{x^{n-1}}+\left.\left(\begin{array}{c}
2 n-2 \\
2
\end{array}\right) D_{2 n-3}^{(1,0, \emptyset, 0)}(x)\right|_{x^{n-2}} \\
& +\frac{28 n^{2}-72 n+39}{24}((2 n-2) ! !) \\
& -\frac{5}{3}\left(\begin{array}{c}
n-1 \\
2
\end{array}\right)((2 n-3) ! !) .
\end{aligned}
$$

Proof. For (1), we proceed by induction on $n$. Now (17) holds for $n=2$ since $\left.A_{4}^{(1,0, \emptyset, 0)}(x)\right|_{x}=2$. Now suppose that $n>2, \sigma=\sigma_{1} \cdots \sigma_{2 n} \in U D_{2 n}$ and $\mathrm{mmp}^{(1,0, \emptyset, 0)}(\sigma)=n-1$. Only $\sigma_{1}, \sigma_{3}, \ldots, \sigma_{2 n-1}$ can match $M M P(1,0, \emptyset, 0)$ in $\sigma$. Now it cannot be that $\sigma_{2 k+1}=1$ where $k<n-2$ since then $\mathrm{mmp}^{(1,0, \emptyset, 0)}(\sigma) \leq k+1<n-1$. Thus, it must be the case that $\sigma_{2 n-3}=1$ or $\sigma_{2 n-1}=1$. Now if $\sigma_{2 n-3}=1$, then $\sigma_{2 n-3}$ matches $M M P(1,0, \emptyset, 0)$ in $\sigma$ and $\sigma_{2 n-1}$ does not match $M M P(1,0, \emptyset, 0)$ in $\sigma$. Then we have $\left(\begin{array}{c}2 n-1 \\ 3\end{array}\right)$ ways to choose the values of $\sigma_{2 n-2}, \sigma_{2 n-1}$, and $\sigma_{2 n}$, and we have two ways to order them. In addition, we must have that $\mathrm{mmp}^{(1,0, \emptyset, 0)}\left(\operatorname{red}\left(\sigma_{1}, \ldots, \sigma_{2 n-4}\right)\right)=$ $n-2$. But then by Theorem 4 , we have $(2 n-5)$ !! ways to choose $\sigma_{1} \cdots \sigma_{2 n-4}$ so that the number of $\sigma=\sigma_{1} \cdots \sigma_{2 n} \in U D_{2 n}$ such that $\sigma_{2 n-3}=1$ and $\mathrm{mmp}^{(1,0, \emptyset, 0)}(\sigma)=n-1$ is

$$
2\left(\begin{array}{c}
2 n-1 \\
3
\end{array}\right)(2 n-5) ! !=\frac{2}{3}(n-1)((2 n-1) ! !) .
$$

Now if $\sigma_{2 n-1}=1$, then we have $2 n-1$ ways to pick the value of $\sigma_{2 n}$ and we must have that $\mathrm{mmp}^{(1,0, \emptyset, 0)}\left(\operatorname{red}\left(\sigma_{1}, \ldots, \sigma_{2 n-2}\right)\right)=n-2$. Thus, we have $\left.A_{2(n-1)}(x)\right|_{x^{n-2}}=\frac{2}{3}\left(\begin{array}{c}n-1 \\ 2\end{array}\right)(2 n-3) ! !$ ways to choose $\sigma_{1} \cdots \sigma_{2 n-2}$. Thus, the number of $\sigma=\sigma_{1} \cdots \sigma_{2 n} \in U D_{2 n}$ such that $\sigma_{2 n-1}=1$ and $\mathrm{mmp}^{(1,0, \emptyset, 0)}(\sigma)=$ $n-1$ is $\frac{2}{3}\left(\begin{array}{c}n-1 \\ 2\end{array}\right)(2 n-1) !$ !. Hence,

$$
\left.A_{2 n}(x)\right|_{x^{n-1}}=\frac{2}{3}(n-1)((2 n-1) ! !)+\frac{2}{3}\left(\begin{array}{c}
n-1 \\
2
\end{array}\right)((2 n-1) ! !)=\frac{2}{3}\left(\begin{array}{c}
n \\
2
\end{array}\right)((2 n-1) ! !) .
$$


Part (2) can be proved by induction in a similar manner. Now (18) holds for $n=2$ since $\left.B_{5}^{(1,0, \emptyset, 0)}(x)\right|_{x}=7$. Now suppose that $n>2, \sigma=$ $\sigma_{1} \cdots \sigma_{2 n+1} \in U D_{2 n+1}$, and $\mathrm{mmp}^{(1,0, \emptyset, 0)}(\sigma)=n-1$. Only $\sigma_{1}, \sigma_{3}, \ldots, \sigma_{2 n-1}$ can match $M M P(1,0, \emptyset, 0)$ in $\sigma$. Again, it cannot be that $\sigma_{2 k+1}=1$ where $k<n-2$ since then $\mathrm{mmp}^{(1,0, \emptyset, 0)}(\sigma) \leq k+1<n-1$. Thus, it must be the case that $\sigma_{2 n-3}=1, \sigma_{2 n-1}=1$, and $\sigma_{2 n+1}=1$. Thus, we have three cases.

Case A. $\sigma_{2 n-3}=1$. Then $\sigma_{2 n-3}$ matches $M M P(1,0, \emptyset, 0)$ in $\sigma$ and $\sigma_{2 n-1}$ does not match $M M P(1,0, \emptyset, 0)$ in $\sigma$. Then we have $\left(\begin{array}{c}2 n \\ 4\end{array}\right)$ ways to choose the values of $\sigma_{2 n-2}, \sigma_{2 n-1}, \sigma_{2 n}$, and $\sigma_{2 n+1}$, and we have 5 ways to order them. In addition, we must have that $\mathrm{mmp}^{(1,0, \emptyset, 0)}\left(\operatorname{red}\left(\sigma_{1}, \ldots, \sigma_{2 n-4}\right)\right)=n-2$. But then by Theorem 4 , we have $(2 n-5)$ !! ways to pick $\sigma_{1} \cdots \sigma_{2 n-4}$ so that the number of $\sigma=\sigma_{1} \cdots \sigma_{2 n} \in U D_{2 n}$ such that $\sigma_{2 n-3}=1$ and $\mathrm{mmp}^{(1,0, \emptyset, 0)}(\sigma)=$ $n-1$ is

$$
5\left(\begin{array}{c}
2 n \\
4
\end{array}\right)(2 n-5) ! !=\frac{5}{6} n(n-1)((2 n-1) ! !)=\frac{5}{3}\left(\begin{array}{c}
n \\
2
\end{array}\right)((2 n-1) ! !) .
$$

Case B. $\sigma_{2 n-1}=1$. Then we have $\left(\begin{array}{c}2 n \\ 2\end{array}\right)$ ways to pick the values of $\sigma_{2 n}$, and $\sigma_{2 n+1}$ and we must have that $\mathrm{mmp}^{(1,0, \emptyset, 0)}\left(\operatorname{red}\left(\sigma_{1}, \ldots, \sigma_{2 n-2}\right)\right)=n-2$. Thus, we have $\left.\left.A_{2(n-1)}(x)\right|_{x^{n-2}}=\frac{2}{3}\left(\begin{array}{c}n-1 \\ 2\end{array}\right)(2 n-3) ! !\right)$ ways to pick $\sigma_{1} \cdots \sigma_{2 n-2}$. Thus, number of $\sigma=\sigma_{1} \cdots \sigma_{2 n} \in U D_{2 n}$ such that $\sigma_{2 n-1}=1$ and $\mathrm{mmp}^{(1,0, \emptyset, 0)}(\sigma)=$ $n-1$ is

$$
\left(\begin{array}{c}
2 n \\
2
\end{array}\right) \frac{2}{3}\left(\begin{array}{c}
n-1 \\
2
\end{array}\right)((2 n-3) ! !)=\frac{2}{3} n\left(\begin{array}{c}
n-1 \\
2
\end{array}\right)((2 n-1) ! !)=2\left(\begin{array}{c}
n \\
3
\end{array}\right)((2 n-1) ! !) .
$$

Case C. $\sigma_{2 n+1}=1$. In this case, $\sigma_{2 n+1}$ does not match $M M P(1,0, \emptyset, 0)$ in $\sigma$ so that we must have that $\mathrm{mmp}^{(1,0, \emptyset, 0)}\left(\operatorname{red}\left(\sigma_{1}, \ldots, \sigma_{2 n}\right)\right)=n-1$. By part (1), we have $\frac{2}{3}\left(\begin{array}{c}n \\ 2\end{array}\right)((2 n-1) ! !)$ ways to choose $\sigma_{1} \cdots \sigma_{2 n}$ in this case.

Thus, it follows that $\left.B_{2 n+1}(x)\right|_{x^{n-1}}=\left(\frac{7}{3}\left(\begin{array}{l}n \\ 2\end{array}\right)+2\left(\begin{array}{l}n \\ 3\end{array}\right)\right)((2 n-1) ! !)$.

Before we can prove part (3), we first need to establish the following lemma.

Lemma 8. For $n \geq 1$,

$$
\left.\bar{D}_{2 n+1}(x)\right|_{x^{n}}=\frac{1}{3}\left(n^{2}-1\right)((2 n) ! !) .
$$

Proof. We proceed by induction on $n$. The lemma holds for $n=1$ since $\bar{D}_{3}^{(1,0, \emptyset, 0)}(x)=2 x^{2}$ so that $\left.\bar{D}_{3}(x)\right|_{x}=0$. Now assume that $n \geq 2$ and $\sigma=$ 
$\sigma_{1} \cdots \sigma_{2 n+1} \in D U_{2 n+1}$, and $\operatorname{mmp}^{(1,0, \emptyset, 0)}(\sigma)+\chi\left(\sigma_{1}=2 n+1\right)=n$. Only $\sigma_{1}, \sigma_{2}, \sigma_{4}, \ldots, \sigma_{2 n}$ can match $\operatorname{MMP}(1,0, \emptyset, 0)$ in $\sigma$. Now it cannot be that $\sigma_{2 k}=1$ where $k \leq n-2$ since then $\operatorname{mmp}^{(1,0, \emptyset, 0)}(\sigma)+\chi\left(\sigma_{1}=2 n+1\right) \leq$ $k+1<n$. Thus, it must be the case that $\sigma_{2 n-2}=1$ or $\sigma_{2 n}=1$. Now if $\sigma_{2 n-2}=1$, then $\sigma_{2 n-2}$ matches $\operatorname{MMP}(1,0, \emptyset, 0)$ in $\sigma$ and $\sigma_{2 n}$ does not match $M M P(1,0, \emptyset, 0)$ in $\sigma$. Then we have $\left(\begin{array}{c}2 n \\ 3\end{array}\right)$ ways to choose the values of $\sigma_{2 n-1}$, $\sigma_{2 n}$, and $\sigma_{2 n+1}$, and we have two ways to order them. In addition, we must have that if $\tau=\operatorname{red}\left(\sigma_{1}, \ldots, \sigma_{2 n-3}\right)$, then $\operatorname{mmp}^{(1,0, \emptyset, 0)}(\tau)+\chi\left(\tau_{1}=2 n-3\right)=$ $n-1$. By Lemma 5 , we then have $(2 n-4)$ !! ways to choose $\sigma_{1} \cdots \sigma_{2 n-3}$ so that the number of $\sigma=\sigma_{1} \cdots \sigma_{2 n+1} \in D U_{2 n+1}$ such that $\sigma_{2 n-2}=1$ and $\operatorname{mmp}^{(1,0, \emptyset, 0)}(\sigma)+\chi\left(\sigma_{1}=2 n+1\right)=n$ is

$$
2\left(\begin{array}{c}
2 n \\
3
\end{array}\right)(2 n-4) ! !=\frac{2 n-1}{3}((2 n) ! !) .
$$

Now if $\sigma_{2 n}=1$, then we have $2 n$ ways to pick the value of $\sigma_{2 n+1}$, and if $\alpha=\operatorname{red}\left(\sigma_{1}, \ldots, \sigma_{2 n-1}\right)$, then $\operatorname{mmp}^{(1,0, \emptyset, 0)}(\alpha)+\chi\left(\alpha_{1}=2 n-1\right)=n-1$. Then we have $\left.\bar{D}_{2(n-1)+1}(x)\right|_{x^{n-1}}=\frac{1}{3}\left((n-1)^{2}-1\right)((2 n-2) ! !)$ ways to choose $\sigma_{1} \cdots \sigma_{2 n-1}$. Thus, number of $\sigma=\sigma_{1} \cdots \sigma_{2 n} \in U D_{2 n}$ such that $\sigma_{2 n-1}=1$ and $\operatorname{mmp}^{(1,0, \emptyset, 0)}(\sigma)+\chi\left(\sigma_{1}=2 n+1\right)=n$ is $\frac{1}{3}\left((n-1)^{2}-1\right)(2 n) !$ !. Hence,

$$
\begin{aligned}
& \left.\bar{D}_{2 n+1}(x)\right|_{x^{n}} \\
& \quad=\frac{2 n-1}{3}((2 n) ! !)+\frac{1}{3}\left((n-1)^{2}-1\right)((2 n) ! !)=\frac{1}{3}\left(n^{2}-1\right)((2 n) ! !) .
\end{aligned}
$$

We prove part (3) by induction. We have that (19) holds for $n=2$ since $\left.D_{5}^{(1,0, \emptyset, 0)}(x)\right|_{x}=9$. Now suppose that $n>2, \sigma=\sigma_{1} \cdots \sigma_{2 n+1} \in D U_{2 n+1}$, and $\operatorname{mmp}^{(1,0, \emptyset, 0)}(\sigma)=n$. Only $\sigma_{1}, \sigma_{2}, \sigma_{4}, \ldots, \sigma_{2 n}$ can match $\operatorname{MMP}(1,0, \emptyset, 0)$ in $\sigma$. It cannot be that $\sigma_{2 k}=1$ where $k \leq n-2$ since then $\operatorname{mmp}^{(1,0, \emptyset, 0)}(\sigma) \leq$ $k+1<n$. Hence, it must be the case that $\sigma_{2 n-2}=1$ or $\sigma_{2 n}=1$. Thus, we have two cases.

Case I. $\sigma_{2 n-2}=1$. Then $\sigma_{2 n-2}$ matches $M M P(1,0, \emptyset, 0)$ in $\sigma$ and $\sigma_{2 n}$ does not match $M M P(1,0, \emptyset, 0)$ in $\sigma$. Then we have two subcases.

Subcase I.a. $2 n+1 \in\left\{\sigma_{2 n-1}, \sigma_{2 n}, \sigma_{2 n+1}\right\}$. In this case, we have $\left(\begin{array}{c}2 n-1 \\ 2\end{array}\right)$ ways to choose the values of the other 2 elements in the set $\left\{\sigma_{2 n-1}, \sigma_{2 n}, \sigma_{2 n+1}\right\}$ and then we have 2 ways to order $\sigma_{2 n-1} \sigma_{2 n} \sigma_{2 n+1}$. Then since $2 n+1 \in$ $\left\{\sigma_{2 n-1}, \sigma_{2 n}, \sigma_{2 n+1}\right\}$, we are guaranteed that $\sigma_{1}$ matches $M M P(1,0, \emptyset, 0)$ in $\sigma$. Thus, when we consider $\tau=\operatorname{red}\left(\sigma_{1} \cdots \sigma_{2 n-3}\right)$, we must have that $\operatorname{mmp}^{(1,0, \emptyset, 0)}(\tau)+\chi\left(\tau_{1}=2 n-3\right)=n-1$. It follows from Lemma 5 that 
we have $(2 n-4)$ !! ways to pick $\sigma_{1} \cdots \sigma_{2 n-3}$. Hence, the $\sigma \in D U_{2 n+1}$ in this subcase contribute $2\left(\begin{array}{c}2 n-1 \\ 2\end{array}\right)(2 n-4) ! !=(2 n-1)((2 n-2) ! !)$ to $\left.D_{2 n+1}^{(1,0, \emptyset, 0)}(x)\right|_{x^{n}}$.

Subcase I.b. $2 n+1 \notin\left\{\sigma_{2 n-1}, \sigma_{2 n}, \sigma_{2 n+1}\right\}$. We then have $\left(\begin{array}{c}2 n-1 \\ 3\end{array}\right)$ ways to choose the values of the elements of $\left\{\sigma_{2 n-1}, \sigma_{2 n}, \sigma_{2 n+1}\right\}$ and 2 ways to order them. Because $2 n+1 \notin\left\{\sigma_{2 n-1}, \sigma_{2 n}, \sigma_{2 n+1}\right\}$, we are not guaranteed that $\sigma_{1}$ matches $\operatorname{MMP}(1,0, \emptyset, 0)$ in $\sigma$. Thus, when we consider $\tau=\operatorname{red}\left(\sigma_{1} \cdots \sigma_{2 n-3}\right)$, we must have that $\mathrm{mmp}^{(1,0, \emptyset, 0)}(\tau)=n-1$. It follows from Theorem 4 that we have $(2 n-4) ! !-(2 n-5) !$ ! ways to pick $\sigma_{1} \cdots \sigma_{2 n-3}$. Thus, the $\sigma \in D U_{2 n+1}$ in this subcase contribute

$$
\begin{aligned}
& 2\left(\begin{array}{c}
2 n-1 \\
3
\end{array}\right)((2 n-4) ! !-(2 n-5) ! !) \\
& \quad=\frac{(2 n-1)(2 n-3)}{3}((2 n-2) ! !)-\frac{2}{3}(n-1)((2 n-1) ! !)
\end{aligned}
$$

to $\left.D_{2 n+1}^{(1,0, \emptyset, 0)}(x)\right|_{x^{n}}$.

Case II. $\sigma_{2 n}=1$. In this case $\sigma_{2 n}$ matches $M M P(1,0, \emptyset, 0)$ in $\sigma$. Then again, we have two subcases.

Subcase II.a. $\sigma_{2 n+1}=2 n+1$. Because $2 n+1=\sigma_{2 n+1}$, we are guaranteed that $\sigma_{1}$ matches $\operatorname{MMP}(1,0, \emptyset, 0)$ in $\sigma$. Thus, when we consider $\tau=$ $\operatorname{red}\left(\sigma_{1} \cdots \sigma_{2 n-1}\right)$, we must have that $\operatorname{mmp}^{(1,0, \emptyset, 0)}(\tau)+\chi\left(\tau_{1}=2 n-1\right)=n-1$. It follows from Lemma 8 that we have $\frac{1}{3}\left((n-1)^{2}-1\right)((2 n-2) !$ !) ways to choose $\sigma_{1} \cdots \sigma_{2 n-3}$. Hence, the $\sigma \in D U_{2 n+1}$ in this subcase contribute $\frac{1}{3}\left((n-1)^{2}-1\right)((2 n-2) ! !)$ to $\left.D_{2 n+1}^{(1,0, \emptyset, 0)}(x)\right|_{x^{n}}$.

Subcase II.b. $2 n+1 \neq \sigma_{2 n+1}$. We then have $(2 n-1)$ ways to choose the value of $\sigma_{2 n+1}$. Because $2 n+1 \neq \sigma_{2 n+1}$, then we are not guaranteed that $\sigma_{1}$ matches $\operatorname{MMP}(1,0, \emptyset, 0)$ in $\sigma$. Thus, when we consider $\tau=\operatorname{red}\left(\sigma_{1} \cdots \sigma_{2 n-1}\right)$, we must have that $\operatorname{mmp}^{(1,0, \emptyset, 0)}(\tau)=n-1$. It then follows by induction that the permutations $\sigma \in D U_{2 n+1}$ in this subcase contribute

$$
\begin{aligned}
(2 n-1) & \left(\left(\sum_{k=1}^{n-1} \frac{(5 k-4) k}{3}((2 k-2) ! !) \prod_{i=k+1}^{n-1}(2 i-1)\right)\right. \\
- & \left.\frac{2}{3}\left(\left(\begin{array}{c}
n-1 \\
2
\end{array}\right)-1\right)((2 n-3) ! !)\right) \\
= & \left(\sum_{k=1}^{n-1} \frac{(5 k-4) k}{3}((2 k-2) ! !) \prod_{i=k+1}^{n}(2 i-1)\right)
\end{aligned}
$$




$$
-\frac{2}{3}\left(\left(\begin{array}{c}
n-1 \\
2
\end{array}\right)-1\right)((2 n-1) ! !)
$$

to $\left.D_{2 n+1}^{(1,0, \emptyset, 0)}(x)\right|_{x^{n}}$.

It follows that

$$
\begin{aligned}
& \left.D_{2 n+1}^{(1,0, \emptyset, 0)}(x)\right|_{x^{n}}=(2 n-1)((2 n-2) ! !)+\frac{1}{3}\left((n-1)^{2}-1\right)((2 n-2) ! !) \\
& +\frac{(2 n-1)(2 n-3)}{3}((2 n-2) ! !)-\frac{2 n-2}{3}((2 n-1) ! !) \\
& +\left(\sum_{k=1}^{n-1} \frac{(5 k-4) k}{3}((2 k-2) ! !) \prod_{i=k+1}^{n}(2 i-1)\right) \\
& -\frac{2}{3}\left(\left(\begin{array}{c}
n-1 \\
2
\end{array}\right)-1\right)((2 n-1) ! !) \\
& =((2 n-2) ! !)\left((2 n-1)+\frac{(2 n-1)(2 n-3)}{3}+\frac{(n-1)^{2}-1}{3}\right) \\
& -((2 n-1) ! !)\left(\frac{2}{3}(n-1)+\frac{2}{3}\left(\left(\begin{array}{c}
n-1 \\
2
\end{array}\right)-1\right)\right) \\
& +\left(\sum_{k=1}^{n-1} \frac{(5 k-4) k}{3}((2 k-2) ! !) \prod_{i=k+1}^{n}(2 i-1)\right) \\
& =\frac{(5 n-4) n}{3}((2 n-2) ! !)-\frac{2}{3}\left(\left(\begin{array}{l}
n \\
2
\end{array}\right)-1\right)((2 n-1) ! !) \\
& +\left(\sum_{k=1}^{n-1} \frac{(5 k-4) k}{3}((2 k-2) ! !) \prod_{i=k+1}^{n}(2 i-1)\right) \\
& =\left(\sum_{k=1}^{n} \frac{(5 k-4) k}{3}((2 k-2) ! !) \prod_{i=k+1}^{n}(2 i-1)\right) \\
& -\frac{2}{3}\left(\left(\begin{array}{l}
n \\
2
\end{array}\right)-1\right)((2 n-1) ! !) \text {. }
\end{aligned}
$$

For part (4), suppose that $\sigma=\sigma_{1} \cdots \sigma_{2 n} \in D U_{2 n}$. Then only $\sigma_{1}, \sigma_{2}, \ldots$, $\sigma_{2 n-2}$ can match $M M P(1,0, \emptyset, 0)$ in $\sigma$. Thus, it cannot be the case that $\sigma_{2 k}=1$ where $k<n-2$ since then $\operatorname{mmp}^{(1,0, \emptyset, 0)}(\sigma) \leq k+1<n-1$. Thus, we must have $1 \in\left\{\sigma_{2 n-4}, \sigma_{2 n-2}, \sigma_{2 n}\right\}$. We then have three cases.

Case 1. $\sigma_{2 n}=1$. In this case, $\sigma_{2 n}$ does not match $\operatorname{MMP}(1,0, \emptyset, 0)$ in $\sigma$. Hence, it must be the case that $\operatorname{mmp}^{(1,0, \emptyset, 0)}\left(\operatorname{red}\left(\sigma_{1} \cdots \sigma_{2 n-1}\right)\right)=n-1$ so that by part $(3)$, we have $\left.D_{2 n-1}^{(1,0, \emptyset, 0)}(x)\right|_{x^{n-1}}$ ways to choose $\sigma_{1} \cdots \sigma_{2 n-1}$. 
Case 2. $\sigma_{2 n-2}=1$. In this case, $\sigma_{2 n-2}$ matches $M M P(1,0, \emptyset, 0)$ in $\sigma$. We then have two subcases.

Subcase 2.1. $\sigma_{2 n-1}=2 n$. In this case, we are guaranteed that $\sigma_{1}$ will match $M M P(1,0, \emptyset, 0)$ in $\sigma$. Thus, if $\tau=\operatorname{red}\left(\sigma_{1} \cdots \sigma_{2 n-3}\right)$, then we must have that $\operatorname{mmp}^{(1,0, \emptyset, 0)}(\tau)+\chi\left(\tau_{1}=2 n-3\right)=n-2$. We then have $(2 n-2)$ ways to choose $\sigma_{2 n}$, and once we have chosen $\sigma_{2 n}$, we have $\frac{2}{3}\left((n-2)^{2}-1\right)((2 n-4) ! !)$ ways to choose $\sigma_{1} \cdots \sigma_{2 n-3}$ by Lemma 8 . Thus, the permutations $\sigma \in D U_{2 n}$ in this case contribute $\frac{2}{3}\left((n-2)^{2}-1\right)((2 n-2)$ !! $)$ to $\left.C_{2 n}(x)\right|_{x^{n-1}}$.

Subcase 2.2. $\sigma_{2 n-1} \neq 2 n$. In this case, we are not guaranteed that $\sigma_{1}$ will match $M M P(1,0, \emptyset, 0)$ in $\sigma$. Thus, if $\tau=\operatorname{red}\left(\sigma_{1} \cdots \sigma_{2 n-3}\right)$, then we must have that $\operatorname{mmp}{ }^{(1,0, \emptyset, 0)}(\tau)=n-2$. We then have $\left(\begin{array}{c}2 n-2 \\ 2\end{array}\right)$ ways to choose $\sigma_{2 n-1}$ and $\sigma_{2 n}$, and once we have chosen $\sigma_{2 n-1}$ and $\sigma_{2 n}$, we have $\left.D_{2 n-3}(x)\right|_{x^{n-2}}$ ways to choose $\sigma_{1} \cdots \sigma_{2 n-3}$. Thus, the permutations $\sigma \in D U_{2 n}$ in this case contribute $\left.\left(\begin{array}{c}2 n-2 \\ 2\end{array}\right) D_{2 n-3}(x)\right|_{x^{n-2}}$ to $\left.C_{2 n}(x)\right|_{x^{n-1}}$.

Case 3. $\sigma_{2 n-4}=1$. In this case, $\sigma_{2 n-4}$ matches $M M P(1,0, \emptyset, 0)$ in $\sigma$, but $\sigma_{2 n-2}$ does not match $M M P(1,0, \emptyset, 0)$ in $\sigma$. Again, we have two subcases.

Subcase 3.1. $2 n \in\left\{\sigma_{2 n-3}, \sigma_{2 n-2}, \sigma_{2 n-1}, \sigma_{2 n}\right\}$. In this case, we are guaranteed that $\sigma_{1}$ will match $M M P(1,0, \emptyset, 0)$ in $\sigma$. Thus, if $\tau=\operatorname{red}\left(\sigma_{1} \cdots \sigma_{2 n-5}\right)$, then we must have that $\operatorname{mmp}^{(1,0, \emptyset, 0)}(\tau)+\chi\left(\tau_{1}=2 n-5\right)=n-2$. We then have $\left(\begin{array}{c}2 n-2 \\ 3\end{array}\right)$ ways to choose the remaining elements for $\left\{\sigma_{2 n-3}, \sigma_{2 n-2}, \sigma_{2 n-1}, \sigma_{2 n}\right\}$. Once we have chosen the remaining elements for $\left\{\sigma_{2 n-3}, \sigma_{2 n-2}, \sigma_{2 n-1}, \sigma_{2 n}\right\}$, we have 5 ways to order them and we have $\left.\bar{D}_{2 n-5}(x)\right|_{x^{n-2}}$ ways to choose $\sigma_{1} \cdots \sigma_{2 n-5}$. By Lemma $5,\left.\bar{D}_{2 n-5}(x)\right|_{x^{n-2}}=(2 n-6)$ !! . Thus, the permutations $\sigma \in D U_{2 n}$ in this case contribute $5\left(\begin{array}{c}2 n-2 \\ 3\end{array}\right)((2 n-6) ! !)=\frac{5}{6}(2 n-3)((2 n-$ $2)$ !!) to $\left.C_{2 n}(x)\right|_{x^{n-1}}$.

Subcase 3.2. $2 n \notin\left\{\sigma_{2 n-3}, \sigma_{2 n-2}, \sigma_{2 n-1}, \sigma_{2 n}\right\}$. In this case, we are not guaranteed that $\sigma_{1}$ will match $M M P(1,0, \emptyset, 0)$ in $\sigma$. Thus, if $\tau=\operatorname{red}\left(\sigma_{1} \cdots \sigma_{2 n-5}\right)$, then we must have that $\operatorname{mmp}^{(1,0, \emptyset, 0)}(\tau)=n-2$. We then have $\left(\begin{array}{c}2 n-2 \\ 4\end{array}\right)$ ways to choose the set $\left\{\sigma_{2 n-3}, \sigma_{2 n-2}, \sigma_{2 n-1}, \sigma_{2 n}\right\}$. Once we have chosen $\left\{\sigma_{2 n-3}, \sigma_{2 n-2}, \sigma_{2 n-1}, \sigma_{2 n}\right\}$, we have 5 ways to order them and we have $\left.D_{2 n-5}(x)\right|_{x^{n-2}}$ ways to choose $\sigma_{1} \cdots \sigma_{2 n-5}$. By Theorem $4,\left.D_{2 n-5}(x)\right|_{x^{n-2}}=$ $(2 n-6) ! !-(2 n-7)$ !!. Thus, the permutations $\sigma \in D U_{2 n}$ in this case contribute

$$
\begin{aligned}
& 5\left(\begin{array}{c}
2 n-2 \\
4
\end{array}\right)((2 n-6) ! !-(2 n-7) ! !) \\
& \quad=\frac{5}{24}(2 n-3)(2 n-5)((2 n-2) ! !)-\frac{5}{3}\left(\begin{array}{c}
n-1 \\
2
\end{array}\right)((2 n-3) ! !)
\end{aligned}
$$


to $\left.C_{2 n}(x)\right|_{x^{n-1}}$.

It follows that

$$
\begin{aligned}
\left.C_{2 n}(x)\right|_{x^{n-1}}= & \left.D_{2 n-1}(x)\right|_{x^{n-1}}+\left.\left(\begin{array}{c}
2 n-2 \\
2
\end{array}\right) D_{2 n-3}(x)\right|_{x^{n-2}} \\
& +\frac{2}{3}\left((n-2)^{2}-1\right)((2 n-2) ! !)+\frac{5}{6}(2 n-3)((2 n-2) ! !) \\
& \times \frac{5}{24}(2 n-3)(2 n-5)((2 n-2) ! !)-\frac{5}{3}\left(\begin{array}{c}
n-1 \\
2
\end{array}\right)((2 n-3) ! !) \\
= & \left.D_{2 n-1}(x)\right|_{x^{n-1}}+\left.\left(\begin{array}{c}
2 n-2 \\
2
\end{array}\right) D_{2 n-3}(x)\right|_{x^{n-2}} \\
& +\left(\frac{28 n^{2}-72 n+39}{24}\right)((2 n-2) ! !)-\frac{5}{3}\left(\begin{array}{c}
n-1 \\
2
\end{array}\right)((2 n-3) ! !) .
\end{aligned}
$$

\section{Conclusions}

As pointed out in [8], the simple type of recursions for the distribution of $\mathrm{mmp}^{(1,0, \emptyset, 0)}(\sigma)$ for $\sigma$ in $U D_{n}$ or $D U_{n}$ proved in this paper no longer holds for the distribution of $\mathrm{mmp}^{(k, 0,0,0)}(\sigma)$ for $\sigma$ in $U D_{n}$ or $D U_{n}$ if $k \geq 2$. For example, suppose that we try to develop a recursion for $A_{2 n}^{(2,0, \emptyset, 0)}(x)=$ $\sum_{\sigma \in U D_{2 n}} x^{\mathrm{mmp}^{(2,0, \emptyset, 0)}(\sigma)}$. Then if we consider the permutations $\sigma=\sigma_{1} \cdots$ $\sigma_{2 n} \in U D_{2 n}$ such that $\sigma_{2 k+1}=1$, we still have $\left(\begin{array}{c}2 n-1 \\ 2 k\end{array}\right)$ ways to pick the elements for $\sigma_{1} \cdots \sigma_{2 k}$. However, in this case the question of whether some $\sigma_{i}$ with $i \leq 2 k$ matches $M M P(2,0, \emptyset, 0)$ in $\sigma$ is dependent on what values occur in $\sigma_{2 k+2} \cdots \sigma_{2 n}$. For example, if $2 n \in\left\{\sigma_{2 k+2}, \ldots, \sigma_{2 n}\right\}$, then every $\sigma_{i}$ with $i \leq k$ will match $\operatorname{MMP}(2,0, \emptyset, 0)$ in $\sigma$. However, if $2 n \in\left\{\sigma_{1}, \ldots, \sigma_{2 k-1}\right\}$, this will not be the case. Thus, we cannot develop a simple recursion for $A_{2 n}^{(2,0, \emptyset, 0)}(x)$.

However, one can develop recursions similar to the ones used in this paper to study the distribution in up-down and down-up permutations of other quadrant marked meshed patterns $\operatorname{MMP}(a, b, c, d)$ in the case where $a, b, c, d \in\{\emptyset, 1\}$. Indeed, in some cases, there are simple relations between such distributions beyond those given in Proposition 1. For example, consider the statistics $\mathrm{mmp}^{(1,0, \emptyset, 0)}(\sigma)$ and $\mathrm{mmp}^{(0,0, \emptyset, 0)}(\sigma)$ over $U D_{2 n}$. Clearly, for any $\sigma=\sigma_{1} \cdots \sigma_{2 n} \in U D_{2 n}, \sigma_{2 i}$ can never match $M M P(1,0, \emptyset, 0)$ or $\operatorname{MMP}(0,0, \emptyset, 0)$ since $\left(2 i-1, \sigma_{2 i-1}\right)$ will always be an element of $G(\sigma)$ that lies in the third quadrant with respect to the coordinate system centered 
at $\left(2 i, \sigma_{2 i}\right)$. On the other hand, elements of the form $\sigma_{2 i-1}$ for $i=1, \ldots, n$, always have an element $G(\sigma)$ in the first quadrant relative to the coordinate system centered at $\left(2 i-1, \sigma_{2 i-1}\right)$, namely $\left(2 i, \sigma_{2 i}\right)$. Thus, if $\sigma \in U D_{2 n}$, then $\mathrm{mmp}^{(1,0, \emptyset, 0)}(\sigma)=\mathrm{mmp}^{(0,0, \emptyset, 0)}(\sigma)$. Therefore, for all $n \geq 1$,

$$
A_{2 n}^{(1,0, \emptyset, 0)}(x)=A_{2 n}^{(0,0, \emptyset, 0)}(x) .
$$

It is not true that $\mathrm{mmp}^{(1,0, \emptyset, 0)}(\sigma)=\mathrm{mmp}^{(0,0, \emptyset, 0)}(\sigma)$ for all $\sigma \in U D_{2 n+1}$ since if $\sigma=\sigma_{1} \cdots \sigma_{2 n+1} \in U D_{2 n+1}$ and $\sigma_{2 n+1}=1$, then $\sigma_{2 n+1}$ matches $M M P(0,0, \emptyset, 0)$ in $\sigma$ but does not match $M M P(1,0, \emptyset, 0)$ in $\sigma$. However, this is the only case where $\mathrm{mmp}^{(1,0, \emptyset, 0)}(\sigma)$ and $\mathrm{mmp}^{(0,0, \emptyset, 0)}(\sigma)$ differ. That is, if $\sigma=\sigma_{1} \cdots \sigma_{2 n+1} \in U D_{2 n+1}$ and $\sigma_{2 n+1} \neq 1$, then $\sigma_{2 n+1}$ does not match $M M P(0,0, \emptyset, 0)$ in $\sigma$ and we can argue as above that $\mathrm{mmp}^{(1,0, \emptyset, 0)}(\sigma)=$ $\mathrm{mmp}^{(0,0, \emptyset, 0)}(\sigma)$. However if $\sigma_{2 n+1}=1$, then $\sigma_{2 n+1}$ matches $M M P(0,0, \emptyset, 0)$ in $\sigma$ but does not match $M M P(1,0, \emptyset, 0)$ in $\sigma$. Thus, if $\sigma \in U D_{2 n+1}^{(2 n+1)}$, $1+\mathrm{mmp}^{(1,0, \emptyset, 0)}(\sigma)=\mathrm{mmp}^{(0,0, \emptyset, 0)}(\sigma)$. It is easy to see that

$$
\sum_{\sigma \in U D_{2 n+1}^{(2 n+1)}} x^{\mathrm{mmp}^{(1,0, \emptyset, 0)}(\sigma)}=A_{2 n}^{(1,0, \emptyset, 0)}(x)
$$

so that for all $n \geq 1$,

$$
B_{2 n+1}^{(0,0, \emptyset, 0)}(x)+(1-x) A_{2 n}^{(1,0, \emptyset, 0)}(x)=B_{2 n+1}^{(1,0, \emptyset, 0)}(x) .
$$

A slightly more subtle relation holds between the distribution of $\mathrm{mmp}^{(1,0,0,0)}(\sigma)$ and $\mathrm{mmp}^{(1,0, \emptyset, 0)}(\sigma)$ for $\sigma \in U D_{2 n}$. For example, in [8], the authors computed the following table for $A_{2 n}^{(1,0,0,0)}(x)$.

\begin{tabular}{|l|l|}
\hline$n$ & $A_{2 n}^{(1,0,0,0)}(x)$ \\
\hline 0 & 1 \\
\hline 1 & $\mathrm{x}$ \\
\hline 2 & $x^{2}(3+2 x)$ \\
\hline 3 & $x^{3}\left(15+30 x+16 x^{2}\right)$ \\
\hline 4 & $x^{4}\left(105+420 x+588 x^{2}+272 x^{3}\right)$ \\
\hline 5 & $x^{5}\left(945+6300 x+16380 x^{2}+18960 x^{3}+7936 x^{4}\right)$ \\
\hline 6 & $x^{6}\left(10395+103950 x+429660 x^{2}+893640 x^{3}+911328 x^{4}+353792 x^{5}\right)$ \\
\hline
\end{tabular}

Comparing the tables for $A_{2 n}^{(1,0,0,0)}(x)$ and $A_{2 n}^{(1,0, \emptyset, 0)}(x)$, one is naturally led to conjecture that for all $n \geq 1$ and $1 \leq k \leq n$,

$$
\left.A_{2 n}^{(1,0, \emptyset, 0)}(x)\right|_{x^{k}}=\left.A_{2 n}^{(1,0,0,0)}(x)\right|_{x^{2 n-k}} .
$$


This follows from comparing $\sigma=\sigma_{1} \cdots \sigma_{2 n} \in U D_{2 n}$ with its reverse complement $\left(\sigma^{r}\right)^{c}=\left(2 n+1-\sigma_{2 n}\right)\left(2 n+1-\sigma_{2 n-1}\right) \cdots\left(2 n+1-\sigma_{1}\right)$ which is also in $U D_{2 n}$. That is, suppose that $\sigma_{i}$ matches $M M P(1,0, \emptyset, 0)$ in $\sigma$. Then $i$ must be odd, i.e. $i=2 k+1$ for some $0 \leq k \leq n-1$, and there must be no elements in $\sigma_{1} \cdots \sigma_{2 k}$ which are less than $\sigma_{2 k+1}$. This means that in $\left(\sigma^{r}\right)^{c},\left(2 n+1-\sigma_{2 k+1}\right)$ has no elements to its right which are greater than $\left(2 n+1-\sigma_{2 k+1}\right)$ so that $\left(2 n+1-\sigma_{2 k+1}\right)$ will not match $\operatorname{MMP}(1,0,0,0)$ in $\left(\sigma^{r}\right)^{c}$. Vice versa, if $\sigma_{2 k+1}$ does not match $M M P(1,0, \emptyset, 0)$ in $\sigma$, there is an element in $\sigma_{1} \cdots \sigma_{2 k}$ which is less than $\sigma_{2 k+1}$. This means that in $\left(\sigma^{r}\right)^{c},\left(2 n+1-\sigma_{2 k+1}\right)$ has an element to its right which is greater than $\left(2 n+1-\sigma_{2 k+1}\right)$ so that $\left(2 n+1-\sigma_{2 k+1}\right)$ will match $M M P(1,0,0,0)$ in $\left(\sigma^{r}\right)^{c}$. Similarly, in $\sigma$, none of $\sigma_{2}, \sigma_{4}, \ldots, \sigma_{2 n}$ will match $M M P(1,0, \emptyset, 0)$ while in $\left(\sigma^{r}\right)^{c}$, each of $\left(2 n+1-\sigma_{2}\right), \ldots,\left(2 n+1-\sigma_{2 n}\right)$ will match $M M P(1,0,0,0)$ in $\left(\sigma^{r}\right)^{c}$. Thus, it follows that for all $\sigma \in U D_{2 n}$,

$$
n+\left(n-\mathrm{mmp}^{(1,0, \emptyset, 0)}(\sigma)\right)=\mathrm{mmp}^{(1,0,0,0)}\left(\left(\sigma^{r}\right)^{c}\right) .
$$

This shows that (22) holds.

There is no such simple relation between the distribution of $\mathrm{mmp}^{(1,0,0,0)}(\sigma)$ and the distribution of $\mathrm{mmp}^{(1,0, \emptyset, 0)}(\sigma)$ for $U D_{2 n+1}, D U_{2 n}$ or $D U_{2 n+1}$ as can be seen from the following tables computed in [8].

Based on these tables, we conjectured in [8] that the polynomials $A_{2 n}^{(1,0,0,0)}(x), B_{2 n+1}^{(1,0,0,0)}(x), C_{2 n}^{(1,0,0,0)}(x)$, and $D_{2 n+1}^{(1,0,0,0)}(x)$ are unimodal for all $n \geq 1$. We also conjecture that $A_{2 n}^{(1,0, \emptyset, 0)}(x), B_{2 n+1}^{(1,0, \emptyset, 0)}(x), C_{2 n}^{(1,0, \emptyset, 0)}(x)$, and $D_{2 n+1}^{(1,0, \emptyset, 0)}(x)$ are unimodal for all $n \geq 1$. Of course, by (22), the conjectures for $A_{2 n}^{(1,0,0,0)}(x)$ and $A_{2 n}^{(1,0, \emptyset, 0)}(x)$ are equivalent.

Finally, we suggest that it should be interesting to study the distribution of quadrant marked mesh patterns on other classes of pattern-restricted permutations such as 2-stack-sortable permutations or vexillary permutations (see [6] for definitions of these) and many other permutation classes having nice properties.

\begin{tabular}{|l|l|}
\hline$n$ & $B_{2 n-1}^{(1,0,0,0)}(x)$ \\
\hline 1 & 1 \\
\hline 2 & $2 x$ \\
\hline 3 & $8 x^{2}(1+x)$ \\
\hline 4 & $16 x^{3}\left(3+8 x+6 x^{2}\right)$ \\
\hline 5 & $128 x^{4}\left(3+15 x+27 x^{2}+17 x^{3}\right)$ \\
\hline 6 & $256 x^{5}\left(15+120 x+381 x^{2}+556 x^{3}+310 x^{4}\right)$ \\
\hline 7 & $1024 x^{6}\left(45+525 x+2562 x^{2}+6420 x^{3}+8146 x^{4}+4146 x^{5}\right)$ \\
\hline
\end{tabular}




\begin{tabular}{|l|l|}
\hline$n$ & $C_{2 n}^{(1,0,0,0)}(x)$ \\
\hline 0 & 1 \\
\hline 1 & 1 \\
\hline 2 & $x(2+3 x)$ \\
\hline 3 & $x^{2}\left(8+28 x+25 x^{2}\right)$ \\
\hline 4 & $x^{3}\left(48+296 x+614 x^{2}+427 x^{3}\right)$ \\
\hline 5 & $x^{4}\left(384+3648 x+13104 x^{2}+20920 x^{3}+12465 x^{4}\right)$ \\
\hline 6 & $x^{5}\left(3840+51840 x+282336 x^{2}+769072 x^{3}+1039946 x^{4}+555731 x^{5}\right)$ \\
\hline
\end{tabular}

\begin{tabular}{|l|l|}
\hline$n$ & $D_{2 n-1}^{(1,0,0,0)}(x)$ \\
\hline 1 & 1 \\
\hline 2 & $x(1+x)$ \\
\hline 3 & $x^{2}\left(3+8 x+5 x^{2}\right)$ \\
\hline 4 & $x^{3}\left(15+75 x+121 x^{2}+61 x^{3}\right)$ \\
\hline 5 & $x^{4}\left(105+840 x+2478 x^{2}+3128 x^{3}+1385 x^{4}\right)$ \\
\hline 6 & $x^{5}\left(945+11025 x+51030 x^{2}+115350 x^{3}+124921 x^{4}+50521 x^{5}\right)$ \\
\hline 7 & $x^{6}\left(10395+166320 x+1105335 x^{2}+3859680 x^{3}+7365633 x^{4}\right.$ \\
& $\left.\quad+7158128 x^{5}+2702765 x^{6}\right)$ \\
\hline
\end{tabular}

\section{Acknowledgments}

We are grateful to the anonymous referee for careful reading of the paper.

\section{References}

[1] D. André (1879). Développements de sec x et de tang x. C. R. Acad. Sci. Paris $\mathbf{8 8}$ 965-967.

[2] D. André (1881). Mémoire sur les permutations alternées. J. Math. Pur. Appl. 7 167-184.

[3] S. Avgustinovich, S. Kitaev and A. Valyuzhenich (2013). Avoidance of boxed mesh patterns on permutations. Discrete Appl. Math. 161 43-51. MR2973348

[4] P. Brändén and A. Claesson (2011). Mesh patterns and the expansion of permutation statistics as sums of permutation patterns. Elect. J. Comb. 18(2), \#P5, 14pp. MR2795782

[5] Í. Hilmarsson, I. Jónsdóttir, S. Sigurdardottir, H. Úlfarsson and S. Vidarsdóttir. Partial Wilf-classification of small mesh patterns, in preparation. 
[6] S. Kitaev (2011). Patterns in Permutations and Words, SpringerVerlag. MR3012380

[7] S. Kitaev and J. Remmel (2012). Quadrant marked mesh patterns. J. Integer Sequences 12 Issue 4, Article 12.4.7. MR2914898

[8] S. Kitaev and J. Remmel (2012). Quadrant marked mesh patterns in alternating permutations. Séminaire Lotharingien de Combinatoire B68a, 20 pp. MR2914898

[9] S. Kitaev, J. Remmel and M. Tiefenbruck. Marked mesh patterns in 132-avoiding permutations I, Pure Mathematics and Applications (Pu.M.A.), special issue on "Permutation Patterns", to appear.

[10] S. Kitaev, J. Remmel and M. Tiefenbruck. Quadrant marked mesh patterns in 132-avoiding permutations II, arXiv:1302.2274. MR2914898

[11] S. Kitaev, J. Remmel and M. Tiefenbruck. Quadrant marked mesh patterns in 132-avoiding permutations III, arXiv:1303.0854. MR2914898

[12] N. J. A. Sloane. The on-line encyclopedia of integer sequences, published electronically at http://www.research.att.com/ njas/ sequences/.

[13] R. P. Stanley (1999). Enumerative Combinatorics, vol. 2, Cambridge University Press. MR1676282

[14] H. Úlfarsson (2011). A unification of permutation patterns related to Schubert varieties, arXiv:1002.4361. MR2924751

Sergey KitaeV

Department of Computer and Information Sciences

UNIVERSITY OF STRATHCLYDE

Livingstone Tower, 26 Richmond Street

Glasgow G1 1XH

United KingDOM

E-mail address: sergey.kitaev@cis.strath.ac.uk

JEFFREY REMMEL

Department of Mathematics

University of California, San Diego

LA Jolla, CA 92093-0112

USA

E-mail address: jremmel@ucsd.edu

ReCeived July 9, 2012 